\title{
NONZERO-SUM STOCHASTIC DIFFERENTIAL GAMES WITH STOPPING TIMES AND FREE BOUNDARY PROBLEMS(')
}

\author{
BY
}

\author{
ALAIN BENSOUSSAN AND AVNER FRIEDMAN
}

\begin{abstract}
One is given a diffusion process and two payoffs which depend on the process and on two stopping times $\tau_{1}, \tau_{2}$. Two players are to choose their respective stopping times $\tau_{1}, \tau_{2}$ so as to achieve a Nash equilibrium point. The problem whether such times exist is reduced to finding a "regular" solution $\left(u_{1}, u_{2}\right)$ of a quasi-variational inequality. Existence of a solution is established in the stationary case and, for one space dimension, in the nonstationary case; for the latter situation, the solution is shown to be regular if the game is of zero sum.
\end{abstract}

Introduction. We consider in this paper a nonzero-sum stochastic differential game with two players, where the decision variables of the players are stopping times. The system evolves according to a stochastic differential equation. The player who decides to stop first prevents the other from continuing and payoffs are computed for each of them. We are looking for a Nash equilibrium point. The model is a natural generalization of the two player zero-sum game studied in Friedman [14]. In Chapter 1 we give sufficient conditions for existence of a Nash equilibrium point. These conditions are stated in terms of functions $u_{1}(x, t), u_{2}(x, t)$ which represent the payoffs for the Nash equilibrium point when the system starts at time $t$ in position $x \in R^{n}$ :

Suppose there exist functions $u_{1}, u_{2}$ satisfying the following system of differential inequalities:

$$
\begin{aligned}
& u_{i}(x, T)=h_{i}(x), \\
& u_{i} \leqslant \phi_{i}, \\
& \text { if } u_{j}=\phi_{j} \text { for } j \neq i \text {, then } u_{i}=\psi_{i}, \\
& \text { if } u_{j}<\phi_{j} \text { for } j \neq i \text {, then }
\end{aligned}
$$

Received by the editors June 13, 1975.

AMS (MOS) subject classifications (1970). Primary 93E05, 49C10; Secondary 35J99, 35K60, 35K99, 60G40, 60H 10, 93E20.

Key words and phrases. Stochastic differential games, stochastic differential equations, stopping time, payoff, Nash point, variational inequality, quasi-variational inequality, free boundary problem, nonzero-sum game, zero-sum game.

(') This work was partially supported by National Science Foundation Grant GP-35347X. 


$$
-\frac{\partial u_{i}}{\partial t}+A(t) u_{i} \leqslant f_{i}
$$

$$
\left(u_{i}-\phi_{i}\right)\left(-\frac{\partial u_{i}}{\partial t}+A(t) u_{i}-f_{i}\right)=0
$$

where $A(t)$ is a second order elliptic differential operator in $x$ and $\phi_{i}, \psi_{i}, f_{i}, h_{i}$ are given functions (in terms of which the game is defined); suppose also that the $u_{i}$ satisfy some regularity conditions. Then there exists a Nash equilibrium point for the initial problem.

In Chapter 2 we study the existence of solutions for $(0.1)-(0.4)$ in the stationary case (i.e., the $u_{i}$ do not depend on $t$ ). We formulate the problem as a quasi-variational inequality, and then prove an existence theorem based upon the study of fixed points of a monotone increasing mapping. The regularity as well as the uniqueness of the solution is left open, the latter being probably false.

In Chapter 3 we study the existence of a solution for $(0.1)-(0.4)$ in the (nonstationary) case $n=1$. Here again an existence theorem is established using a fixed point theorem for a monotone increasing mapping. Under suitable conditions it is proved that the free boundary consists of two monotone and continuous curves $x=s_{i}(t), s_{1}(t)<s_{2}(t)$.

The fixed point theorem used in Chapters 2, 3 is due to Tartar [23]. It was also applied in [20] in order to solve a parabolic quasi-variational inequality arising from a Stefan problem of melting of ice with variable latent heat.

In Chapters 4, 5 we use the method of nonlinear Volterra integral equations in order to solve parabolic free boundary problems in one space dimension. In Chapter 4 we consider a Stefan type problem involving two "temperatures" $\theta_{1}$ and $\theta_{2}$. By classical methods ([12], [13]) one establishes the existence of a unique regular solution as long as $\theta_{1 x}, \theta_{2 x}$ are a priori bounded. When the initial "temperatures" are nonnegative, a priori bounds can be obtained without difficulty (see the proof of Theorem 4.1). When the initial "temperatures" take also negative values, it is harder to establish the necessary a priori estimates (see proofs of Theorems 4.3, 4.4). Our results in this case are extensions of the works of Van Moerbeke [24] and Friedman [16].

We also derive in Chapter 4 an estimate on $s_{2}(t)-s_{1}(t)$ as $t \rightarrow \infty$.

In Chapter 5 we apply the methods of Chapter 4 to a somewhat different system of Stefan type problem which corresponds to the zero-sum game (in the case $n=1$ ); this system is in some sense a special case of the system studied in Chapter 4. We deduce that not only are the functions $u_{1}(x, t)$ and $u_{2}(x, t)$ regular (which is already known for general $n \geqslant 1$, by Friedman [14]) but also the free boundaries are regular curves. 
Chapter 1. The Differential Game With Stopping Times and Characterization of a Nash Point by a System of Differential INEQUALITIES

1.1. Assumptions; notation. Let $(\Omega, Q, P)$ be a probability space and $\mathcal{T}_{t}$, $t \geqslant 0$, be an increasing family of sub $\sigma$-algebras of $\mathcal{Q}$. Let $b(t)$ be an $n$-dimensional (standard) Brownian motion with respect to the family $\mathscr{F}_{t}$. Let $g(x, t)$ be an $n$-vector defined on $R^{n} \times[0, \infty)$ and let $\sigma(x, t)$ be an $n \times n$ matrix defined on $R^{n} \times[0, \infty)$, such that

(1.1) $g$ is continuous and bounded,

(1.2) $\left|g(x, t)-g\left(x^{\prime}, t\right)\right| \leqslant C\left|x-x^{\prime}\right|$ for all $x, x^{\prime} \quad$ (C constant),

(1.3) $\sigma$ is continuous and bounded,

(1.4) $\partial \sigma(x, t) / \partial x$ is measurable and bounded,

(1.5) $\sigma^{-1}$ is continuous and bounded.

We denote by $y_{x t}(s), s \geqslant t$, the solution of the stochastic differential equation

$$
d y_{x t}(s)=g\left(y_{x t}(s), s\right) d s+\sigma\left(y_{x t}(s), s\right) d b(s), \quad y_{x t}(t)=x .
$$

In the following all the stopping times which we use are considered to be stopping times with respect to $\mathscr{F}_{t}$.

Let $T$ be a positive number and let $f_{i}(x, t), \phi_{i}(x, t), \psi_{i}(x, t)(i=1,2)$ be functions such that

$$
\begin{aligned}
& f_{i}, \phi_{i}, \psi_{i} \text { are continuous and bounded } \\
& \text { in } R^{n} \times[0, T], f_{i} \in L^{2}\left(R^{n} \times(0, T)\right), \\
& \psi_{i} \leqslant \phi_{i} \text { for all } x, t \text { in } R^{n} \times[0, T] \\
& (i=1,2) .
\end{aligned}
$$

1.2. Definition of a Nash point in the nonstationary case. We consider two players such that each of them may decide to stop the process $y_{x t}$ starting at time $t \leqslant T$. Given two functions $h_{1}(x), h_{2}(x)$ satisfying

$$
h_{i} \text { are continuous and bounded, }
$$

we define the payoff functions $J_{x t}^{i}\left(\tau_{1}, \tau_{2}\right)$, where $\tau_{i}$ are stopping times such that $t \leqslant \tau_{i} \leqslant T$, as follows:

$$
\begin{aligned}
J_{x t}^{i}\left(\tau_{1}, \tau_{2}\right)=E\left[\int_{t}^{\tau_{1} \wedge \tau_{2}} f_{i}\left(y_{x t}(s), s\right) d s+\chi_{\tau_{i}<\tau_{j}} \phi_{i}\left(y_{x t}\left(\tau_{i}\right), \tau_{i}\right)\right. & \\
& \left.+\chi_{\tau_{i}>\tau_{j}, T>\tau_{j}} \psi_{i}\left(y_{x t}\left(\tau_{j}\right), \tau_{j}\right)+\chi_{\tau_{1}=\tau_{2}=T} h_{i}\left(y_{x t}(T)\right)\right] \\
& (j \neq i)
\end{aligned}
$$

where $\chi_{A}$ is the indicator function of $A$.

Our problem is to find a Nash point for the $J_{x t}^{i}$, i.e., to find $\hat{\tau}_{1}, \hat{\tau}_{2}$ such that 
(1.10) $J_{x t}^{1}\left(\hat{\tau}_{1}, \hat{\tau}_{2}\right) \leqslant J_{x t}^{1}\left(\tau_{1}, \hat{\tau}_{2}\right), \quad J_{x t}^{2}\left(\hat{\tau}_{1}, \hat{\tau}_{2}\right) \leqslant J_{x t}^{2}\left(\hat{\tau}_{1}, \tau_{2}\right)$ for any $\tau_{1}, \tau_{2}$.

1.3. System of differential inequalities. For $t$ fixed, $t \in[0, T]$, we define the second order differential operator $A(t)$ by

$$
A(t) w=-\sum_{i, j=1}^{n} \frac{1}{2} a_{i j}(x, t) \frac{\partial^{2} w}{\partial x_{i} \partial x_{j}}-\sum_{j=1}^{n} g_{j}(x, t) \frac{\partial w}{\partial x_{j}}
$$

where the $a_{i j}(x, t)$ are the components of the matrix $a(x, t)=\sigma \sigma^{*}(x, t)$, $\sigma^{*}=$ transpose of $\sigma$, and $g_{j}(x, t)$ are the components of $g(x, t)$. Let $Q=R^{n}$ $\times(0, T)$.

We introduce the following problem: Find two functions $u_{1}(x, t), u_{2}(x, t)$ such that

(1.12) $u_{i}$ is continuous and bounded in $\bar{Q}, u_{i} \in L^{2}\left(0, T ; H^{1}\left(R^{n}\right)\right)$;

(1.13) $\quad u_{i}(x, T)=h_{i}(x) \quad\left(x \in R^{n}\right)$;

(1.14) $\quad u_{i}(x, t) \leqslant \phi_{i}(x, t)$ in $Q$

(1.15) if $u_{j}(x, t)=\phi_{j}(x, t)$ for $j \neq i$ and some $(x, t)$ in $Q$,

then $u_{i}(x, t)=\psi_{i}(x, t)$

if $\Sigma_{i}=\left\{(x, t) \in Q ; u_{j}(x, t)<\phi_{j}(x, t)\right.$ for $\left.j \neq i\right\}$, then

$$
\begin{aligned}
& \frac{\partial u_{i}}{\partial t}-A(t) u_{i} \in L^{2}\left(\Sigma_{i}\right), \quad-\frac{\partial u_{i}}{\partial t}+A(t) u_{i} \leqslant f_{i} \quad \text { a.e. in } \Sigma_{i} \\
& \left(u_{i}-\phi_{i}\right)\left[-\frac{\partial u_{i}}{\partial t}+A(t) u_{i}-f_{i}\right]=0 \quad \text { a.e. in } \Sigma_{i} .
\end{aligned}
$$

We will refer to (1.12)-(1.16) as the system of differential inequalities (associated with the stochastic differential game).

To $u_{i}$ we correspond a set $C_{i}$ defined by

$$
C_{i}=\left\{(x, t) \in Q ; u_{i}(x, t)<\phi_{i}(x, t)\right\}
$$

which by virtue of the continuity properties of the functions $u_{i}$ and $\phi_{i}$ is an open subset of $Q$. (Notice that $C_{1}=\Sigma_{2}, C_{2}=\Sigma_{1}$.)

1.4. Sufficiency criterion for existence of a Nash point. We define the exit time of $C_{i}$ by

$$
\begin{aligned}
\hat{\tau}_{i} & =\inf \left\{s ; t<\leqslant T, y_{x t}(s) \notin C_{i}\right\} \quad \text { if such numbers } s \text { exist, } \\
& =T \text { otherwise. }
\end{aligned}
$$

THEOREM 1.1. Let the assumptions (1.1)-(1.5), (1.7) and (1.8) hold. If there exist functions $u_{1}, u_{2}$ satisfying (1.12)-(1.16), then for any $x, t$, the $\hat{\tau}_{i}$ given by (1.18) form a Nash point for the payoff functions $J_{x t}^{i}$. Furthermore,

$$
u_{i}(x, t)=J_{x t}^{i}\left(\hat{\tau}_{1}, \hat{\tau}_{2}\right) \text {. }
$$


Proof. We shall use the following slight extension of Ito's formula. Let

$$
\Phi(x, t) \in L^{2}\left(0, T ; H^{1}\left(R^{n}\right)\right), \quad\left[\frac{\partial \Phi}{\partial t}-A(t) \Phi\right] \in L^{2}(Q),
$$

$\Phi$ continuous and bounded on $\bar{Q}$. Then for any stopping time $\theta, t \leqslant \theta \leqslant T$, and for any $\varepsilon>0$ we have

$$
\begin{aligned}
E \Phi\left(y_{x t}(\theta), \theta\right)= & E \Phi\left(y_{x t}(\theta \wedge(t+\varepsilon)), \theta \wedge(t+\varepsilon)\right) \\
& +E \int_{\theta \wedge(t+\varepsilon)}^{\theta}\left(\frac{\partial \Phi}{\partial t}-A(t) \Phi\right)\left(y_{x t}(s), s\right) d s .
\end{aligned}
$$

For the proof of (1.20) see [3], [4]. Let us note that $\hat{\tau}_{j}(j \neq i)$ is the exit time of $\Sigma_{i}$. If we restrict ourselves to stopping times $\theta$ such that $t \leqslant \theta \leqslant \hat{\tau}_{j}$, then we may apply (1.20) with functions $\Phi$ such that

$$
\Phi \in L^{2}\left(0, T ; H^{1}\left(R^{n}\right)\right), \quad\left[\frac{\partial \Phi}{\partial t}-A(t) \Phi\right] \in L^{2}\left(\Sigma_{i}\right) .
$$

Indeed, for any $\lambda>0$ let $\Sigma^{\lambda}=\left\{(x, t) \in \Sigma_{i}\right.$, dist $\left.\left((x, t), \partial \Sigma_{i}\right)>\lambda\right\}, \gamma_{\lambda}=$ exit time from $\Sigma^{\lambda}$. Since we can modify $\Phi$ outside $\Sigma^{\lambda}$ so that the modified function satisfies the regularity properties of $(1.20)$, we conclude that $(1.20)$ is valid with $\theta$ replaced by $\theta \wedge \gamma_{\lambda}$. Taking $\lambda \downarrow 0,(1.20)$ follows.

Let $t \leqslant \tau_{1}, \tau_{2} \leqslant T$ be stopping times. We shall prove that

$$
u_{1}(x, t) \leqslant J_{x t}^{1}\left(\tau_{1}, \hat{\tau}_{2}\right), \quad u_{2}(x, t) \leqslant J_{x t}^{2}\left(\hat{\tau}_{1}, \tau_{2}\right) .
$$

Let $j=1$ if $i=2$ and $j=2$ if $i=1$. Applying (1.20) with $\Phi=u_{i}$ and $\theta=\tau_{i} \wedge \hat{\tau}_{j}$, we get

$$
\begin{aligned}
E u_{i}\left(y_{x t}\left(\tau_{i} \wedge \hat{\tau}_{j}\right), \tau_{i} \wedge \hat{\tau}_{j}\right) & \\
= & E u_{i}\left(y_{x t}\left(\tau_{i} \wedge \hat{\tau}_{j} \wedge(t+\varepsilon)\right), \tau_{i} \wedge \hat{\tau}_{j} \wedge(t+\varepsilon)\right) \\
& +E \int_{\tau_{i} \wedge \hat{\tau}_{j} \wedge(t+\varepsilon)}^{\tau_{i} \wedge \hat{\tau}_{j}}\left(\frac{\partial u_{i}}{\partial t}+A(t) u_{i}\right)\left(y_{x t}(s), s\right) d s .
\end{aligned}
$$

From (1.16) it follows that

$$
\begin{aligned}
E \int_{\tau_{i} \wedge \hat{\tau}_{j} \wedge(t+\varepsilon)}^{\tau_{i} \wedge \hat{j}_{j}}( & \left.-\frac{\partial u_{i}}{\partial t}+A(t) u_{i}\right)\left(y_{x t}(s), s\right) d s \\
& <E \int_{\tau_{i} \wedge \hat{j}_{j} \wedge(t+\varepsilon)}^{\tau_{i} \wedge \hat{\tau}_{j}} f_{i}\left(y_{x t}(s), s\right) d s
\end{aligned}
$$

From (1.13) and (1.14) we also have

$$
\begin{aligned}
& u_{i}\left(y_{x t}\left(\tau_{i} \wedge \hat{\tau}_{j}\right), \tau_{i} \wedge \hat{\tau}_{j}\right) \leqslant \phi_{i}\left(y_{x t}\left(\tau_{i}\right), \tau_{i}\right) \chi_{\tau_{i}<\hat{\tau}_{j}} \\
& \quad+\chi_{\tau_{i}>\hat{j}_{j}, T>\hat{\tau}_{j}} \psi_{i}\left(y_{x t}\left(\hat{\tau}_{j}\right), \hat{\tau}_{j}\right)+\chi_{\tau_{i}=\hat{j}_{j}=T} h_{i}\left(y_{x t}(T)\right) .
\end{aligned}
$$


Using (1.23), (1.24) in (1.22), letting $\varepsilon \rightarrow 0$ and noting that

$$
E u_{i}\left(y_{x t}\left(\tau_{i} \wedge \hat{\tau}_{j} \wedge(t+\varepsilon), \tau_{i} \wedge \hat{\tau}_{j} \wedge(t+\varepsilon)\right)\right) \rightarrow u_{i}(x, t)
$$

since $u_{i}$ is continuous and bounded, we obtain (1.21).

It remains to prove that

$$
u_{i}(x, t)=J_{x t}^{i}\left(\hat{\tau}_{1}, \hat{\tau}_{2}\right)
$$

We use similar arguments to those above.

From (1.16) we get, if $j \neq i$,

$$
\begin{gathered}
E \int_{\hat{\tau}_{i} \wedge \hat{\tau}_{j} \wedge(t+\varepsilon)}^{\hat{\tau}_{i} \wedge \hat{\tau}_{j}}\left(-\frac{\partial u_{i}}{\partial t}+A(t) u_{i}\right)\left(y_{x t}(s), s\right) d s \\
=E \int_{\hat{\tau}_{i} \wedge \hat{\tau}_{j}}^{\hat{\tau}_{i} \wedge \hat{\tau}_{j}} f_{i}\left(y_{x t}(s), s\right) d s .
\end{gathered}
$$

From (1.13), (1.14) and the fact that if $\hat{\tau}_{i}<\hat{\tau}_{j}$ then

$$
u_{i}\left(y_{x t}\left(\hat{\tau}_{i}\right), \hat{\tau}_{i}\right)=\phi_{i}\left(y_{x t}\left(\hat{\tau}_{i}\right), \hat{\tau}_{i}\right)
$$

we get (1.24) with equality instead of inequality and $\hat{\tau}_{i}$ instead of $\tau_{i}$. Taking $\tau_{i}=\hat{\tau}_{i}$ in (1.22), using (1.26) and (1.24) (with equality), and letting $\varepsilon \rightarrow 0$, we obtain (1.25), which completes the proof of the theorem.

\subsection{Stationary case. We now assume that}

$$
g, \sigma, f_{i}, \phi_{i}, \psi_{i} \text { do not depend on } t ; T=+\infty \text {. }
$$

We introduce a constant (discount factor) $\alpha>0$ and define the payoff

$$
\begin{aligned}
J_{x}^{i}\left(\tau_{1}, \tau_{2}\right)=E\left[\int_{0}^{\tau_{1} \wedge \tau_{2}} e^{-\alpha t} f_{i}\left(y_{x}(t)\right) d t\right. & +\psi_{\tau_{i}<\tau_{j}} \phi_{i}\left(y_{x}\left(\tau_{i}\right)\right) e^{-\alpha \tau_{i}} \\
& \left.+\chi_{\tau_{i}>\tau_{j}} \psi_{i}\left(y_{x}\left(\tau_{j}\right)\right) e^{-\alpha \tau_{j}}\right] \\
& (j \neq i),
\end{aligned}
$$

where $y_{x}(t)$ denotes $y_{x 0}(t)$. As in $\S 1.3$, we introduce functions $u_{1}(x), u_{2}(x)$ (not depending on time) such that

(1.29) $\quad u_{i}$ is continuous and bounded, $u_{i} \in H^{1}\left(R^{n}\right)$;

(1.30) $u_{i} \leqslant \phi_{i} \quad\left(x \in R^{n}\right)$;

(1.31) if $u_{j}(x)=\phi_{j}(x)$ for $j \neq i$ and some $x$, then $u_{i}(x)=\psi_{i}(x)$;

if $\Sigma_{i}=\left\{x \in R^{n} ; u_{j}(x)<\phi_{j}(x)\right\}(j \neq i)$, then

(1.32) $A u_{i} \in L^{2}\left(\Sigma_{i}\right), \quad A u_{i} \leqslant f_{i}$ a.e. in $\Sigma_{i}$,

$$
\left(u_{i}-\phi_{i}\right)\left(A u_{i}-f_{i}\right)=0 \text { a.e. in } \Sigma_{i} \text {. }
$$

Here, 


$$
A w=-\frac{1}{2} \sum_{i, j=1}^{n} a_{i j}(x) \frac{\partial^{2} w}{\partial x_{i} \partial x_{j}}-\sum_{i=1}^{n} g_{i}(x) \frac{\partial w}{\partial x_{i}}+\alpha w
$$

where $\left(a_{i j}\right)$ is the matrix $\sigma \sigma^{*}(x)$ and $g_{i}(x)$ are the components of $g$. We define

$$
C_{i}=\left\{x \in R^{n} ; u_{i}(x)<\phi_{i}(x)\right\}
$$

and state without proof a theorem analogous to Theorem 1.1:

THEOREM 1.2. Under the assumptions of Theorem 1.1 and (1.27), if there exist functions $u_{1}(x), u_{2}(x)$ satisfying (1.29)-(1.32), and if $\hat{\tau}_{i}=\inf \left\{s>0, y_{x}(s) \notin\right.$ $\left.C_{i}\right\}$, then the pair $\left(\hat{\tau}_{1}, \hat{\tau}_{2}\right)$ forms a Nash point for the payoff functions (1.28). Furthermore,

$$
u_{i}(x)=J_{x}^{i}\left(\hat{\tau}_{1}, \hat{\tau}_{2}\right)
$$

It is assumed here that $\hat{\tau}_{1}, \hat{\tau}_{2}$ are well defined, i.e., that $y_{x}(s) \notin C_{i}$ for some time $s=s_{i}(i=1,2)$.

ReMARK. The results of this chapter can be extended to $N$-person nonzerosum game with payoffs

$$
\begin{aligned}
& J_{x t}^{i}\left(\tau_{1}, \ldots, \tau_{N}\right)=E\left[\int_{t}^{\tau_{1} \wedge \cdots \wedge \tau_{N}} f_{i}\left(y_{x t}(s), s\right) d s+\chi_{\tau_{i}<\wedge \wedge \neq t \tau_{j}} \phi_{i}\left(y_{x t}\left(\tau_{i}\right), \tau_{i}\right)\right. \\
& +\chi_{\tau_{i}>\wedge_{j \neq i} \tau_{j}, T>\wedge_{j \neq i} \tau_{j}} \psi_{i}\left(y_{x t}\left(\bigwedge_{j \neq i} \tau_{j}\right), \bigwedge_{j \neq i} \tau_{j}\right) \\
& \left.+\chi_{\tau_{1}}=\cdots=\tau_{N}=T h_{i}\left(y_{x t}(T)\right)\right] .
\end{aligned}
$$

In the condition (1.15) one has to assume that $u_{j}(x, t)=\phi_{j}(x, t)$ for some $j \neq i$, and in the definition of $\Sigma_{i}$ in $(1.16) u_{j}(x, t)<\phi_{j}(x, t)$ for all $j \neq i$. A Nash equilibrium point $\left(\hat{\tau}_{1}, \ldots, \hat{\tau}_{N}\right)$ is defined by

$$
J_{i}\left(\hat{\tau}_{1}, \ldots, \hat{\tau}_{N}\right) \leqslant J_{i}\left(\hat{\tau}_{1}, \ldots, \hat{\tau}_{i-1}, \tau_{i}, \hat{\tau}_{i+1}, \ldots, \hat{\tau}_{N}\right) \quad(1 \leqslant i \leqslant N)
$$

for any $\tau_{1}, \ldots, \tau_{N}$. Theorems $1.1,1.2$ and their proofs readily extend to this case.

\section{Chapter 2. Stationary Quasi-Variational INEQualities}

In this Chapter we consider the stationary case for any dimension $n$.

2.1. Assumptions; notation. Consider a second order differential operator

$$
A w=-\frac{1}{2} \sum_{i, j=1}^{n} \frac{\partial}{\partial x_{i}}\left(a_{i j} \frac{\partial w}{\partial x_{j}}\right)+\sum_{j=1}^{n} a_{j} \frac{\partial w}{\partial x_{j}}+a_{0} w
$$

where

$$
\left\{\begin{array}{l}
a_{i j}(x), a_{j}(x) \text { are functions in } L^{\infty}\left(R^{n}\right) \\
a_{0}(x) \geqslant \alpha>0 ; a_{i j}=a_{j i} .
\end{array}\right.
$$


When

$$
a_{j}(x)=\frac{1}{2} \sum_{i} \frac{\partial a_{i j}}{\partial x_{i}}-g_{j}(x), \quad a_{0}(x)=\alpha,
$$

we get the operator $A$ of (1.33). We shall make the assumptions:

$$
\sum_{i, j} a_{i j} \xi_{j} \xi_{i} \geqslant \gamma \sum_{i} \xi_{i}^{2} \text { for all real } \xi_{i}, \gamma \text { positive constant, }
$$

$$
\left.a_{j} \text { is differentiable and } a_{0}(x)-\frac{1}{2} \sum_{j} \frac{\partial a_{j}}{\partial x_{j}} \geqslant \beta>0 \quad \text { ( } \beta \text { constant }\right) .
$$

Let $V=H^{1}\left(R^{n}\right)$. We define on the Hilbert space $V$ a bilinear continuous form $a(u, v)$ :

$$
a(u, v)=\frac{1}{2} \sum_{i, j} \int_{R^{n}} a_{i j} \frac{\partial u}{\partial x_{j}} \frac{\partial v}{\partial x_{i}} d x+\sum_{j} \int_{R^{n}} a_{j} \frac{\partial u}{\partial x_{j}} v d x+\int_{R^{n}} a_{0} u v d x .
$$

Noting that

$$
\begin{aligned}
a(u) & =a(u, u)=\frac{1}{2} \sum_{i, j} \int_{R^{n}} a_{i j} \frac{\partial u}{\partial x_{j}} \frac{\partial u}{\partial x_{i}} d x+\int_{R^{n}}\left(a_{0}-\frac{1}{2} \sum_{j} \frac{\partial a_{j}}{\partial x_{j}}\right) u^{2} d x \\
& \geqslant \beta \int_{R^{n}} u^{2} d x
\end{aligned}
$$

we conclude that

$$
a(u)=0 \text { implies } u=0 .
$$

We consider functions $f_{i}, \phi_{i}, \psi_{i}(i=1,2)$ satisfying (1.7) and (1.27). For $u_{1}, u_{2}$ in $L^{2}\left(R^{n}\right)$ define the sets

$$
\begin{aligned}
K_{i}\left(u_{j}\right)=\left\{v \in V ; v \leqslant \phi_{i} \text { a.e. and a.e. in } x,\right. \\
\text { if } \left.u_{j}(x) \geqslant \phi_{j}(x) \text { then } v(x)=\psi_{i}(x)\right\} \quad(j \neq i) .
\end{aligned}
$$

Clearly $K_{i}\left(u_{j}\right)$ is a closed convex subset of $V$. It is not empty since $v=\psi_{i} \in$ $K_{i}\left(u_{j}\right)$. We denote by $($,$) the scalar product in L^{2}\left(R^{n}\right)$ and by $(()$,$) the scalar$ product in $V$.

2.2. System of Q.V.I. We consider the following problem: find $u_{1}, u_{2}$ in $V$ such that

$$
\begin{array}{ll}
u_{1} \in K_{1}\left(u_{2}\right), & u_{2} \in K_{2}\left(u_{1}\right), \\
a\left(u_{1}, v-u_{1}\right) \geqslant\left(f_{1}, v-u_{1}\right) & \text { for every } v \in K_{1}\left(u_{2}\right), \\
a\left(u_{2}, v-u_{2}\right) \geqslant\left(f_{2}, v-u_{2}\right) & \text { for every } v \in K_{2}\left(u_{1}\right) .
\end{array}
$$

We call (2.10) a system of quasi-variational inequalities (Q.V.I.). We can formulate (2.10) as one Q.V.I. in $V^{2}=V \times V$, defining for $u=\left(u_{1}, u_{2}\right)$, $v=\left(v_{1}, v_{2}\right)$, the bilinear form 


$$
\tilde{a}(u, v)=\sum_{i=1}^{2} a\left(u_{i}, v_{i}\right)
$$

and setting $\tilde{f}=\left(f_{1}, f_{2}\right)$,

$$
K(u)=\left\{v=\left(v_{1}, v_{2}\right) ; v_{1} \in K_{1}\left(v_{2}\right), v_{2} \in K_{2}\left(v_{1}\right)\right\}
$$

With this notation (2.10) is equivalent to

$$
\tilde{a}(u, v-u) \geqslant(\tilde{f}, v-u) \text { for every } v \in K(u), u \in K(u) .
$$

In $(2.11)$ by $(\tilde{f}, v-u)$ we mean $\sum_{i=1}^{2}\left(f_{i}, v_{i}-u_{i}\right)$.

Now (2.11) is a Q.V.I. as defined in [2], [4], [5], [6]. Systems of Q.V.I. have already been considered by Bensoussan-Lions [7]. However, the situation here differs from the one considered by these authors, because the convex set $K(u)$ is decreasing with $u$ instead of increasing. Let us state this more precisely. We consider in $V^{2}$ the natural order relationship

$$
v \geqslant v^{\prime} \text { if } v_{i}(x) \geqslant v_{i}^{\prime}(x) \text { a.e., for } i=1,2 .
$$

One easily checks that

$$
K(v) \subset K\left(v^{\prime}\right) \text { if } v \geqslant v^{\prime}
$$

We say that the convex set decreases, and that the Q.V.I. (2.11) is a decreasing Q.V.I. For such Q.V.I. the general existence results of Bensoussan-Lions [7] and of Tartar [23] cannot be applied. Decreasing Q.V.I. have already been considered by Bensoussan-Lions [8], but with assumptions on the continuity of $K(v)$ with respect to $v$, which are not satisfied here.

Before giving existence results for (2.10), we shall show how (2.10) is related to (1.29)-(1.32). We have

THEOREM 2.1. Let (2.3) hold. Suppose there exists a solution $\left(u_{1}, u_{2}\right)$ of $(2.10)$ such that the functions $u_{i}$ are continuous and bounded. Define $\Sigma_{i}$ as in (1.32) and suppose that $\Sigma_{i}$ has a smooth boundary, that the complement of $\Sigma_{i}$ is not of measure 0, and that $A u_{i} \in L^{2}\left(\Sigma_{i}\right)$. Then the $u_{i}$ 's form a solution of (1.29)-(1.32).

Proof. Let $S_{i}$ be the complement of $\Sigma_{i}$. Since $u_{i} \in K_{i}\left(u_{j}\right)(j \neq i)$, we have $u_{i}(x)=\psi_{i}(x)$ a.e. in $S_{i}$. By continuity we have $u_{i}=\psi_{i}$ everywhere in $S_{i}$, which proves (1.31).

Noting that if $v \in K_{i}\left(u_{j}\right), j \neq i$, then $v=u_{i}$ on $S_{i}$ and we get from (2.10)

$$
\begin{gathered}
\frac{1}{2} \int_{\Sigma_{i}}\left[\sum_{k, l=1}^{n} a_{k l} \frac{\partial u_{i}}{\partial x_{l}} \frac{\partial\left(v-u_{i}\right)}{\partial x_{k}}+\sum_{k=1}^{n} a_{k} \frac{\partial u_{i}}{\partial x_{k}}\left(v-u_{i}\right)+a_{0} u_{i}\left(v-u_{i}\right)\right] d x \\
\geqslant \int_{\Sigma_{i}} f_{i}\left(v-u_{i}\right) d x
\end{gathered}
$$

Integrating by parts in the first integral and noting that $v=u_{i}$ on $\partial \Sigma_{i}$ we get, since $A u_{i} \in L^{2}\left(\Sigma_{i}\right)$, 


$$
\int_{\Sigma_{i}}\left(A u_{i}-f_{i}\right)\left(v-u_{i}\right) d x \geqslant 0 \text { for any } v \in K_{i}\left(u_{j}\right)(j \neq i) .
$$

Let $\theta$ be an open ball such that $\theta$ is contained in $\Sigma_{i}$. Then we can find a family of smooth functions $\theta_{\varepsilon}, 0 \leqslant \theta_{\varepsilon} \leqslant 1$, such that $\theta_{\varepsilon}=0$ outside $\Sigma_{i}$ and $\theta_{\varepsilon}(x) \rightarrow 1$ in $\theta, \theta_{\varepsilon} \rightarrow 0$ outside $\theta$ if $\varepsilon \rightarrow 0$. We may take in (2.15) $v=u_{i}-\theta_{\varepsilon}, \varepsilon$ small, which is admissible. We then get

$$
\int_{\theta} \theta_{\varepsilon}\left(A u_{i}-f_{i}\right) d x+\int_{\Sigma_{i}-\theta} \theta_{\varepsilon}\left(A u_{i}-f_{i}\right) d x \leqslant 0 .
$$

Letting $\varepsilon \downarrow 0$, we obtain $\int_{\mathcal{O}}\left(A u_{i}-f_{i}\right) d x \leqslant 0$, and since $\mathcal{\theta}$ is arbitrary,

$$
A u_{i}-f_{i} \leqslant 0 \text { a.e. in } \Sigma_{i} \text {. }
$$

Now let $\theta$ be as above and take in (2.15) the admissible function $v=\phi_{i} \theta_{e}$ $+\left(1-\theta_{\varepsilon}\right) u_{i}$. Then

$$
\begin{aligned}
& \int_{\Theta}\left(A u_{i}-f_{i}\right)\left[\left(\phi_{i} \theta_{\varepsilon}-u_{i}\right)+\left(1-\theta_{\varepsilon}\right) u_{i}\right] d x \\
& \quad+\int_{\Sigma_{i}-\Theta}\left(A u_{i}-f_{i}\right)\left[\phi_{i} \theta_{\varepsilon}-\theta_{\varepsilon} u_{i}\right] d x \geqslant 0 .
\end{aligned}
$$

Letting $\varepsilon \downarrow 0$, we get $\int_{0}\left(A u_{i}-f_{i}\right)\left(\phi_{i}-u_{i}\right) d x \geqslant 0$. But from (2.16) and $u_{i} \leqslant \phi_{i}$, we also have the reverse inequality. Hence

$$
\int_{0}\left(A u_{i}-f_{i}\right)\left(\phi_{i}-u_{i}\right) d x=0 .
$$

Since again $\theta$ is arbitrary, the last relation in (1.32) follows. We have thereby completed the proof of (1.29)-(1.32).

Remark. Theorem 2.1 extends to the case of $N$-person game. In the definition (2.9), $K_{i}\left(u_{j}\right)$ should be replaced by $K_{i}\left(u_{1}, \ldots, u_{i-1}, u_{i+1}, \ldots, u_{N}\right)$ and the assertion $v(x)=\psi_{i}(x)$ a.e. is required to follow from $u_{j}(x) \geqslant \phi_{j}(x)$ for some $j \neq i$.

2.3. Existence theorem. We shall now give an existence rsult for a system of Q.V.I. (2.10), making the following restrictive assumptions:

$$
\begin{aligned}
& f_{i}, \phi_{i}, \psi_{i} \text { are continuous and bounded; } \\
& f \in L^{2}\left(R^{n}\right), \phi_{i} \in V, \psi_{i} \in V, \psi_{i} \leqslant \phi_{i}, \\
& \text { and } a\left(\psi_{i}, \chi\right)-\left(f_{i}, \chi\right) \leqslant 0 \text { for any } \\
& \chi \in V, \chi \geqslant 0(i=1,2) .
\end{aligned}
$$

Notice that the last inequality holds if $\psi_{i} \in H^{2}\left(R^{n}\right)$ and $A \psi_{i} \leqslant f_{i}$.

THEOREM 2.2. Let the assumptions (2.2)-(2.5), (2.18) hold. Then there exists a solution $u_{1}, u_{2}$ of the Q.V.I. (2.10) satisfying $u_{i} \geqslant \psi_{i}$ for $i=1$, 2. Moreover there exist two pairs of solutions $\left(\underline{u}_{1}, \bar{u}_{2}\right)$ and $\left(\bar{u}_{1}, \underline{u}_{2}\right)$ such that if $u_{1}, u_{2}$ is a solution then

$$
\psi_{1} \leqslant \underline{u}_{1} \leqslant u_{1} \leqslant \bar{u}_{1} \leqslant \phi_{1}, \quad \psi_{2} \leqslant \underline{u}_{2} \leqslant u_{2} \leqslant \bar{u}_{2} \leqslant \phi_{2} .
$$


Proof. We shall define several mappings. Let $w_{1}, w_{2} \in L^{2}\left(R^{n}\right)$. Recall that

$$
\begin{aligned}
& K_{2}\left(w_{1}\right)=\left\{v \in V ; v \leqslant \phi_{2} \text { a.e. and a.e. if } w_{1} \geqslant \phi_{1} \text { then } v=\psi_{2}\right\}, \\
& K_{1}\left(w_{2}\right)=\left\{v \in V ; v \leqslant \phi_{1} \text { a.e. and a.e. if } w_{2} \geqslant \phi_{2} \text { then } v=\psi_{1}\right\} .
\end{aligned}
$$

In $L^{2}\left(R^{n}\right)$ we define a mapping $T_{2}$ as follows: for a given $w_{1} \in L^{2}\left(R^{n}\right)$, $u_{2}=T_{2} w_{1}$ is the solution of the variational inequality

$$
a\left(u_{2}, v-u_{2}\right) \geqslant\left(f_{2}, v-u_{2}\right) \text { for any } v \in K_{2}\left(w_{1}\right), u_{2} \in K_{2}\left(w_{1}\right) .
$$

The solution of (2.21) exists and is unique (cf. [21]).

Next we define a mapping $T_{1}$ : for a given $w_{2} \in L^{2}\left(R^{n}\right), u_{1}=T_{1} w_{2}$ is the solution of the variational inequality

$$
a\left(u_{1}, v-u_{1}\right) \geqslant\left(f_{1}, v-u_{1}\right) \text { for all } v \in K_{1}\left(w_{2}\right), u_{1} \in K_{1}\left(w_{2}\right) .
$$

We define a mapping $S_{1}$ in $L^{2}\left(R^{n}\right)$ by $S_{1}=T_{1} T_{2}$, i.e.,

$$
u_{1}=S_{1} w_{1}=T_{1} T_{2} w_{1} \text {. }
$$

We also define $S_{2}=T_{2} T_{1}$. It is clear that if $u_{1}$ is a fixed point for $S_{1}$, then $\left(u_{1}\right.$, $T_{2} u_{1}$ ) is a solution of the Q.V.I.; further, $T_{2} u_{1}$ is then a fixed point for $S_{2}$ (and, conversely, if $u_{2}$ is a fixed point for $S_{2}$, then $T_{1} u_{2}$ is a fixed point for $S_{1}$, $\left(T_{1} u_{2}, u_{2}\right)$ is a solution of the Q.V.I., and $T_{1} u_{2}$ is a fixed point for $\left.S_{1}\right)$.

The crucial fact to be proved below is that

$$
\begin{aligned}
& S_{1} \text { (resp. } S_{2} \text { ) is increasing in the sense } \\
& \text { that if } w_{1} \leqslant w_{1}^{\prime} \text { a.e. (resp. } w_{2} \leqslant w_{2}^{\prime} \text { a.e.) } \\
& \text { then } S_{1} w_{1} \leqslant S_{1} w_{1}^{\prime} \text { a.e. (resp. } S_{2} w_{2} \leqslant \\
& S_{2} w_{2}^{\prime} \text { a.e.). }
\end{aligned}
$$

We shall first verify that

$$
u_{2}=T_{2} w_{1} \geqslant \psi_{2} \text { for all } w_{1}, \quad u_{1}=T_{1} w_{2} \geqslant \psi_{1} \text { for all } w_{2} \text {. }
$$

We note that since $\psi_{1} \in K_{1}\left(w_{2}\right), \max \left(\psi_{1}, u_{1}\right) \in K_{1}\left(w_{2}\right)$. Using $v=\max \left(\psi_{1}, u_{1}\right)$ as a test function in (2.22) and noting that $\max \left(\psi_{1}, u_{1}\right)=u_{1}+\left(u_{1}-\psi_{1}\right)^{-}$, we get

or

$$
a\left(u_{1},\left(u_{1}-\psi_{1}\right)^{-}\right) \geqslant\left(f_{1},\left(u_{1}-\psi_{1}\right)^{-}\right),
$$

$$
a\left(\left(u_{1}-\psi_{1}\right)^{-}\right)-a\left(\psi_{1},\left(u_{1}-\psi_{1}\right)^{-}\right)+\left(f_{1},\left(u_{1}-\psi_{1}\right)^{-}\right) \leqslant 0,
$$

which with (2.18) implies $a\left(\left(u_{1}-\psi_{1}\right)^{-}\right)=0$; hence by (2.8), $u_{1} \geqslant \psi_{1}$. A similar argument shows that $u_{2} \geqslant \psi_{2}$.

Now let $w_{1} \leqslant w_{1}^{\prime}$, and write $u_{2}=T_{2} w_{1}$ and $u_{1}=T_{1} T_{2} w_{1}=S_{1} w_{1}$ (similarly define $u_{2}^{\prime}$ and $u_{1}^{\prime}$ for $\left.w_{1}^{\prime}\right)$. We shall first prove that

$$
u_{2} \geqslant u_{2}^{\prime}
$$

Since $w_{1} \leqslant w_{1}^{\prime}$, we have $K_{2}\left(w_{1}^{\prime}\right) \subset K_{2}\left(w_{1}\right)$. Therefore $u_{2}, u_{2}^{\prime} \in K_{2}\left(w_{1}\right)$, and also $\max \left(u_{2}, u_{2}^{\prime}\right) \in K_{2}\left(w_{1}\right)$. Now from $u_{2}^{\prime} \in K_{2}\left(w_{1}^{\prime}\right)$ it follows that if $w_{1}^{\prime}>\phi_{1}$, 
$\min \left(u_{2}, u_{2}^{\prime}\right) \leqslant u_{2}^{\prime}=\psi_{2}$. But from $(2.25)$ we also have $\min \left(u_{2}, u_{2}^{\prime}\right) \geqslant \psi_{2}$; hence $\min \left(u_{2}, u_{2}^{\prime}\right)=\psi_{2}$ if $w_{1}^{\prime} \geqslant \phi_{1}$. Since $\min \left(u_{2}, u_{2}^{\prime}\right) \leqslant u_{2}^{\prime} \leqslant \phi_{2}$, it follows that $\min \left(u_{2}, u_{2}^{\prime}\right) \in K_{2}\left(w_{1}^{\prime}\right)$. We can thus take $\max \left(u_{2}, u_{2}^{\prime}\right)$ as a test function in (2.21) and $\min \left(u_{2}, u_{2}^{\prime}\right)$ as a test function in $(2.21)^{\prime}$ (i.e. (2.21) for $\left.w_{1}^{\prime}\right)$. Noting that

$$
\max \left(u_{2}, u_{2}^{\prime}\right)=u_{2}+\left(u_{2}^{\prime}-u_{2}\right)^{+}, \quad \min \left(u_{2}, u_{2}^{\prime}\right)=u_{2}^{\prime}-\left(u_{2}^{\prime}-u_{2}\right)^{+},
$$

we get

$$
\begin{gathered}
a\left(u_{2},\left(u_{2}^{\prime}-u_{2}\right)^{+}\right) \geqslant\left(f_{2},\left(u_{2}^{\prime}-u_{2}\right)^{+}\right), \\
-a\left(u_{2}^{\prime},\left(u_{2}^{\prime}-u_{2}\right)^{+}\right) \geqslant-\left(f_{2},\left(u_{2}^{\prime}-u_{2}\right)^{+}\right)
\end{gathered}
$$

and, by addition, $a\left(\left(u_{2}^{\prime}-u_{2}\right)^{+}\right) \leqslant 0$ which with $(2.8)$ implies $\left(u_{2}^{\prime}-u_{2}\right)^{+}=0$; hence (2.26). Now since $u_{2} \geqslant u_{2}^{\prime}$, we have $K_{1}\left(u_{2}\right) \subset K_{1}\left(u_{2}^{\prime}\right)$. Therefore $u_{1}$ and $u_{1}^{\prime} \in K_{1}\left(u_{2}^{\prime}\right)$; hence $\max \left(u_{1}, u_{1}^{\prime}\right) \in K_{1}\left(u_{2}^{\prime}\right)$. Taking (2.25) into account, we prove by a similar argument as above that $\min \left(u_{1}, u_{1}^{\prime}\right) \in K_{1}\left(u_{2}\right)$. We use $\max \left(u_{1}, u_{1}^{\prime}\right)\left(\right.$ resp. $\left.\min \left(u_{1}, u_{1}^{\prime}\right)\right)$ as a test function in the variational inequality for $u_{1}^{\prime}$ (resp. $u_{1}$ ) and, by addition, we obtain $u_{1} \leqslant u_{1}^{\prime}$, which proves (2.24). From (2.25) it follows that

$$
S_{1} \psi_{1} \geqslant \psi_{1} \text {, }
$$

i.e., $\psi_{1}$ is a lower solutior of the equation $S_{1} u_{1}=u_{1}$ in the terminology of Tartar [23]. By construction of $S_{1}$ and $T_{1}$,

$$
S_{1} \phi_{1} \leqslant \phi_{1} \text {, }
$$

i.e., $\phi_{1}$ is an upper solution. Since $\psi_{1} \leqslant \phi_{1}$, we can use a general theorem of Tartar [23], which asserts that $S_{1}$ has a fixed point between $\psi_{1}$ and $\phi_{1}$. Moreover there exists a maximal and a minimal solution. In other words, there exist $\underline{u}_{1}$ and $\bar{u}_{1}$ such that

$$
\begin{aligned}
& \psi_{1} \leqslant \underline{u}_{1} \leqslant \bar{u}_{1} \leqslant \phi_{1}, S_{1} \underline{u}_{1}=\underline{u}_{1}, S_{1} \bar{u}_{1}= \\
& \bar{u}_{1}, \text { and if } u_{1}=S_{1} u_{1}, \psi_{1} \leqslant u_{1} \leqslant \phi_{1} \\
& \text { then } \underline{u}_{1} \leqslant u_{1} \leqslant \bar{u}_{1} .
\end{aligned}
$$

Defining $\bar{u}_{2}=T_{2} \underline{u}_{1}$ and $\underline{u}_{2}=T_{2} \bar{u}_{1}$, we see that $\left(\underline{u}_{1}, \bar{u}_{2}\right)$ and $\left(\bar{u}_{1}, \underline{u}_{2}\right)$ are two pairs of solutions for the Q.V.I.

Let now $u_{1}, u_{2}$ be a solution. By $(2.25), \psi_{1} \leqslant u_{1} \leqslant \phi_{1}$ (and $\psi_{2} \leqslant u_{2} \leqslant \phi_{2}$ ). We thus have $u_{1}=S_{1} u_{1}$ and, according to (2.29), $\underline{u}_{1} \leqslant u_{1} \leqslant \bar{u}_{1}$. Therefore $T_{2} \bar{u}_{1} \leqslant u_{2} \leqslant T_{2} \underline{u}_{1}$ which proves (2.19) and completes the proof of the theorem.

\section{Chapter 3. Parabolic Quasi-Variational Inequalities in One Space Dimension}

3.1. The problem. In this chapter we shall solve the following problem: Find functions $u_{1}(x, t), u_{2}(x, t)$ and curves $s_{1}(t), s_{2}(t)$ with 


$$
s_{1}(0)=-1, \quad s_{2}(0)=1, \quad s_{1}(t)<s_{2}(t) \quad(0<t \leqslant T)
$$

such that

$$
\begin{aligned}
& u_{1 t}-u_{1 x x} \leqslant \tilde{f}_{1} \text { and } u_{1}=\tilde{\phi}_{1} \text { if } x<s_{1}(t), 0<t<T \\
& u_{1 t}-u_{1 x x}=\tilde{f}_{1} \text { and } u_{1}<\tilde{\phi}_{1} \text { if } s_{1}(t)<x<s_{2}(t), 0<t<T \\
& u_{1}=\tilde{\psi}_{1} \text { if } x>s_{2}(t), 0<t<T \\
& u_{1}(x, 0)=h_{1}(x) \text { if }-1<x<1 \\
& \quad u_{2 t}-u_{2 x x} \leqslant \tilde{f}_{2} \text { and } u_{2}=\tilde{\phi}_{2} \text { if } x>s_{2}(t), 0<t<T \\
& u_{2 t}-u_{2 x x}=\tilde{f}_{2} \text { and } u_{2}<\tilde{\phi}_{2} \text { if } s_{1}(t)<x<s_{2}(t), 0<t<T, \\
& u_{2}=\tilde{\psi}_{2} \text { if } x<s_{1}(t), 0<t<T \\
& u_{2}(x, 0)=h_{2}(x) \text { if }-1<x<1 .
\end{aligned}
$$

This system is a parabolic quasi-variational inequality in one space dimension. It can, in fact, be easily reformulated in the more standard Q.V.I. terminology, using convex cones $K_{1}\left(u_{2}(\cdot, t)\right), K_{2}\left(u_{1}(\cdot, t)\right)$.

Assume now that

$$
\begin{aligned}
& h_{i}(x)>0 \quad \text { if }-1<x<1(i=1,2), \\
& h_{1}(x)=0 \quad \text { if } x<-1, \\
& h_{2}(x)=0 \quad \text { if } x>1, \\
& \sigma(x, t) \equiv \sqrt{2}, \quad g(x, t) \equiv 0, \\
& f_{i}(x, t)=\tilde{f}_{i}(x, T-t), \quad \phi_{i}(x, t)=\tilde{\phi}_{i}(x, T-t), \\
& \psi_{i}(x, t)=\tilde{\psi}_{i}(x, T-t), \\
& \psi_{i}(x, t)<\phi_{i}(x, t) \quad(i=1,2) .
\end{aligned}
$$

Suppose there exists a sufficiently "strong" solution of (3.1), (3.2) in the sense that

$$
\begin{aligned}
& u_{i t}, u_{i x}, u_{i x x} \text { are in } L^{2} \text { and } u_{i} \text { is continuous in the region } \\
& s_{1}(t) \leqslant x \leqslant s_{2}(t), 0 \leqslant t \leqslant T .
\end{aligned}
$$

From Theorem 1.1 we then deduce that there exists a Nash equilibrium point $\left(\tau_{1}^{*}, \tau_{2}^{*}\right)$ with $\tau_{i}^{*}$ being the exit time from the set

$$
C_{i}=\left\{(x, t) ; u_{i}(x, t)<\tilde{\phi}_{i}(x, t)\right\} .
$$

In this chapter we shall prove the existence of a "weak" solution of (3.1), (3.2). It will be shown, in fact, that there exists a "maximal" solution $\left(\bar{u}_{1}, \bar{u}_{2}\right)$ and a "minimal" solution $\left(\underline{u}_{1}, \underline{u}_{2}\right)$, i.e., for any other solution $\left(u_{1}, u_{2}\right)$, $\underline{u}_{1} \leqslant u_{1} \leqslant \bar{u}_{1}, \underline{u}_{2} \geqslant u_{2} \geqslant \bar{u}_{2}$, where the functions are defined.

Set 


$$
\gamma_{i}=\tilde{\phi}_{i}-\tilde{\psi}_{i}, \quad k_{i}(x)=\tilde{\phi}_{i}(x, 0)-h_{i}(x) .
$$

Throughout this chapter it is always assumed that

$\gamma_{i t}, \gamma_{i x}, \gamma_{i x x}$ are continuous and bounded for $(x, t) \in R^{1} \times[0, T]$,

$k_{i x}$ are continuous for $x \in R^{1}$,

$k_{1 x x}$ is uniformly continuous for $-1<x<\infty$,

$k_{2 x x}$ is uniformly continuous for $-\infty<x<1$,

$\gamma_{i}(x, t)>0$ for all $(x, t)$,

$k_{i}(x)>0$ if $-1<x<1$,

$k_{1}(x)=0 \quad$ if $x<-1, \quad k_{1}(x)=\gamma_{1}(x, 0)$ if $x>1$,

$k_{2}(x)=\gamma_{2}(x, 0)$ if $x<-1, \quad k_{2}(x)=0$ if $x>1$,

and that

$\tilde{\phi}_{i t}, \tilde{\phi}_{i x}, \tilde{\phi}_{i x x}$ are continuous, $\quad \tilde{\phi}_{i t}-\tilde{\phi}_{i x x}-\tilde{f}_{i} \equiv-1$.

The last condition implies, of course, that $u_{i t}-u_{i x x} \leqslant \tilde{f}_{i}$ a.e. on the set $u_{i}=\tilde{\phi}_{i}$. Notice also that if $u_{1}=\tilde{\psi}_{1}$ on the curve $x=s_{2}(t)$, then we can always define $u_{1}$ for $x>s_{2}(t)$ by $u_{1}=\tilde{\psi}_{1}$. A similar remark applies to $u_{2}$. Hence, setting

$$
w_{1}=\tilde{\phi}_{1}-u_{1}, \quad w_{2}=\tilde{\phi}_{2}-u_{2},
$$

the system (3.1), (3.2) reduces to

$$
\begin{array}{ll}
w_{1 t}-w_{1 x x}=-1 \text { and } w_{1}>0 & \text { if } s_{1}(t)<x<s_{2}(t), 0<t<T, \\
w_{1}=0 & \text { if } x<s_{1}(t), 0<t<T, \\
w_{1}=\gamma_{1} & \text { if } x=s_{2}(t), 0<t<T, \\
w_{1}(x, 0)=k_{1}(x) & \text { if }-1<x<1 ; \\
w_{2 t}-w_{2 x x}=-1 \text { and } w_{2}>0 & \text { if } s_{1}(t)<x<s_{2}(t), 0<t<T, \\
w_{2}=0 & \text { if } x>s_{2}(t), 0<t<T, \\
w_{2}=\gamma_{2} & \text { if } x=s_{1}(t), 0<t<T, \\
w_{2}(x, 0)=k_{2}(x) & \text { if }-1<x<1 .
\end{array}
$$

We shall prove later on the existence of a "weak" solution of (3.6), (3.7) with $s_{1}(t), s_{2}(t)$ (the free boundary curves) having the following properties:

$s_{1}(t)$ is continuous and strictly monotone decreasing,

$s_{2}(t)$ is continuous and strictly monotone increasing.

We outline the idea of the proof: Given a monotone curve $x=s_{2}(t)$, we solve the variational inequality (3.6) and show that its free boundary is a curve $x=\sigma_{1}(t)$. Next we solve the variational inequality (3.7) with $s_{1}(t)$ replaced by $\sigma_{1}(t)$, and show that its free boundary is a curve $x=\bar{s}_{2}(t)$. We have thus constructed a mapping $W, \bar{s}_{2}=W s_{2}$. It will be shown (using 
Tartar's fixed point theorem [23]) that $W$ has fixed points; these points represent solutions of the Q.V.I. (3.6), (3.7). Some technical difficulties arise due to the fact that the monotone curves $s_{2}$ on which $W$ is defined may not be continuous.

3.2. Auxiliary variational inequality. Let $s(t)$ be a monotone increasing function with Hölder continuous first derivative $\dot{s}(t)$, for $0 \leqslant t \leqslant T$, such that $s(0)=1$. Set

$$
G=\{(x, t) ;-\infty<x<s(t), 0<t<T\} .
$$

Consider the variational inequality: Find $w$ such that

$$
\begin{aligned}
& w \geqslant 0 \quad \text { if } x<s(t), 0<t<T, \\
& w_{t}, w_{x}, w_{x x} \text { are in } L^{2}(G) \text {, } \\
& \int_{-\infty}^{s(t)}\left[w_{t}(x, t)-w_{x x}(x, t)\right][v(x)-w(x, t)] d x \\
& \geqslant-\int_{-\infty}^{s(t)}[v(x)-w(x, t)] d x \\
& \text { for a.a. } t \in(0, T) \text {, for any } v \in L^{\infty}\left(R^{1}\right), v \geqslant 0 \text { a.e., } \\
& w=\gamma_{1} \quad \text { if } x=s(t), 0 \leqslant t \leqslant T, \\
& w(x, 0)=k_{1}(x) \text { if } x<1 \text {. }
\end{aligned}
$$

LEMMA 3.1. There exists a unique solution $w$ of (3.9) with compact support; further, $w_{t}, w_{x}, w_{x x}$ belong to $L^{p}(G)$ for any $1<p<\infty$.

FIRST PROOF. Let $\beta_{\varepsilon}(t)$ be a family of $C^{\infty}$ functions of $t(-\infty<t<\infty$, $0<\varepsilon<1$ ) such that

$$
\begin{gathered}
\beta_{\varepsilon}(t) \leqslant 0, \quad \beta_{\varepsilon}(0)=-1, \quad \beta_{\varepsilon}^{\prime}(t) \geqslant 0, \\
\beta_{\varepsilon}(t) \rightarrow 0 \quad \text { if } t>0, \varepsilon \rightarrow 0, \quad \beta_{\varepsilon}(t) \rightarrow-\infty \quad \text { if } t<0, \varepsilon \rightarrow 0 .
\end{gathered}
$$

Set $G_{R}=G \cap\{x ; x>-R\}$ for any $R>1$, and consider the parabolic problem

$$
\begin{array}{ll}
w_{t}-w_{x x}+\beta_{\varepsilon}(w)=-1 & \text { in } G_{R}, \\
w(x, 0)=k_{1}(x) & \text { if }-R<x<1, \\
w(s(t), t)=\gamma_{1}(s(t), t) & \text { if } 0<t<T, \\
w(-R, t)=0 & \text { if } 0<t<T .
\end{array}
$$

Denote the solution by $w=w_{\varepsilon, R}$. One can show (cf. [9], [17], [19]) that

$$
-1 \leqslant \beta_{\varepsilon}(w) \leqslant 0 \text {. }
$$

\section{Hence}




$$
\left|w_{t}-w_{x x}\right| \leqslant 1
$$

By the $L^{p}$ estimates for parabolic equations (see, for instance, [2z]) we then deduce that

$$
\iint_{G_{R}}\left[\left|w_{t}\right|^{p}+\left|w_{x}\right|^{p}+\left|w_{x x}\right|^{p}\right] d x d t \leqslant C \quad(1<p<\infty)
$$

where $C$ is a constant independent of $\varepsilon$. Actually, in the $L^{p}$ estimates one usually assumes that the domain $G_{R}$ is cylindrical, i.e., $s(t) \equiv$ const. Therefore in order to obtain (3.13) we first perform a transformation

$$
y=\frac{x}{s(t)}, \quad \tilde{w}(y, t)=w(x, t)
$$

and note that

$$
w_{t}-w_{x x}=\tilde{w}_{t}-\frac{y \dot{s}(t)}{s(t)} \tilde{w}_{y}-\frac{1}{s^{2}(t)} \tilde{w}_{y y} .
$$

We then apply the interior $L^{p}$ estimates to $w(y, t)$ in some region $-\tilde{R}<y<$ $1,0<t<T$. This yields the $L^{p}$ estimates (3.13) in the region $G^{\prime}=\{0<x$ $<s(t), 0<t<T\}$, provided $\tilde{R}$ was suitably chosen, depending on the function $s$. (We assume that $R>\tilde{R}$.) We also have, by interior $L^{p}$ estimates, the estimate (3.13) in $G_{R}-G^{\prime}$. Combining these estimates, (3.13) follows.

Recall that in (3.13), $w$ stands for $w_{\varepsilon, R}$. Taking $\varepsilon \downarrow 0$ through such a sequence that $w_{\varepsilon, R}$ is weakly convergent in the norm $L^{p}\left(G_{R}\right)$ together with the derivatives $\partial / \partial t, \partial / \partial x, \partial^{2} / \partial x^{2}$, we find that the weak limit $w_{R} \equiv \lim w_{\varepsilon, R}$ in the unique solution of the variational inequality

$$
\begin{aligned}
& w \geqslant 0 \text { in } G_{R} \\
& w_{t}, w_{x}, w_{x x} \text { are in } L^{p}\left(G_{R}\right), \quad 1<p<\infty \\
& \int_{-R}^{s(t)}\left(w_{t}-w_{x x}\right)(v-w) d x \geqslant-\int_{-R}^{s(t)}(v-w) d x \text { a.e. in } t \in[0, T], \\
& w=\gamma_{1} \text { if } x=s(t), 0<t<T, \\
& w(x, 0)=k_{1}(x) \text { if }-R<x<1, \\
& w(-R, t)=0 \text { if } 0<t<T .
\end{aligned}
$$

By [10], there exists a number $R_{0}$ sufficiently large such that if $R>R_{0}$ then

$$
w \equiv w_{R}=0 \text { if }-R<x<-R_{0}, 0<t<T .
$$

Hence, by uniqueness,

$$
w_{R^{\prime}}(x, t)=w_{R}(x, t) \text { if }-R<x<s(t), 0<t<T, R^{\prime}>R .
$$

It follows that $w=\lim w_{R}$ is the asserted unique solution of (3.9).

SECOND PROOF. Performing the transformation (3.14), and using (3.15), the 
variational inequality (3.9) reduces to the variational inequality:

$$
\begin{aligned}
& \tilde{w} \geqslant 0 \quad \text { in } \tilde{G} \\
& \tilde{w}_{t}, \tilde{w}_{y}, \tilde{w}_{y y} \text { are in } L^{2}(\tilde{G}), \\
& \int_{-\infty}^{1}\left[\tilde{w}_{t}-\frac{1}{s^{2}(t)} \tilde{w}_{y y}-\frac{y \dot{s}(t)}{s(t)} \tilde{w}_{y}\right](v-\tilde{w}) d y \geqslant-\int_{-\infty}^{1}(v-\tilde{w}) d y
\end{aligned}
$$

for a.a. $t \in(0, T)$, for any $v \in L^{\infty}\left(R^{1}\right), v \geqslant 0$ a.e.,

$$
\begin{aligned}
& \tilde{w}(1, t)=\gamma_{1}(s(t), t) \quad \text { if } 0<t<T, \\
& \tilde{w}(y, 0)=k_{1}(y) \text { if } y<1,
\end{aligned}
$$

where $\tilde{G}=\{(y, t) ;-\infty<y<1,0<t<T\}$.

Let $\tilde{G}_{R}=\tilde{G} \cap\{y, y>-R\}$ and consider the parabolic problem:

$$
\begin{array}{ll}
\tilde{w}_{t}-\frac{1}{s^{2}(t)} \tilde{w}_{y y}-\frac{y \dot{s}(t)}{s(t)} \tilde{w}_{y}+\beta_{\varepsilon}(w)=-1 & \text { in } \tilde{G}_{R}, \\
\tilde{w}(1, t)=\gamma_{1}(s(t), t) & \text { if } 0<t<T, \\
\tilde{w}(y, 0)=k_{1}(y) & \text { if }-R<y<1, \\
\tilde{w}(-R, t)=0 & \text { if } 0<t<T .
\end{array}
$$

Denote its solution by $\tilde{w}=\tilde{w}_{\varepsilon, R}$. Since the coefficients of the parabolic operator in (3.18) are Hölder continuous, the $L^{p}$ parabolic estimates (cf. (3.13)) are valid. We can now proceed as in the first proof and show that when $\varepsilon \downarrow 0$ in a suitable way, the solutions $\tilde{w}_{\varepsilon, R}$ converge weakly in $L^{p}\left(\tilde{G}_{R}\right)$ to a function $\tilde{w}_{R}$ (together with the derivatives $\partial / \partial t, \partial / \partial x, \partial^{2} / \partial x^{2}$ ), and $\tilde{w}_{R}$ is a solution of the variational inequality obtained from (3.17) by replacing $\tilde{G}$ by $\tilde{G}_{R}, \int_{-\infty}^{1}$ by $\int_{-R}^{1}$ and by replacing the last condition in (3.17) by the last two conditions of (3.18).

The techniques of [10] show that the support of $\tilde{w}_{R}$ remains bounded as $R$ increases to infinity. Hence $\tilde{w}_{R^{\prime}}=\tilde{w}_{R}$ in $\tilde{G}_{R}$ if $R^{\prime}>R, R$ sufficiently large. It follows that $\tilde{w}=\lim \tilde{w}_{R}$ is the desired solution.

Remark 1. From the first proof, $w=\lim _{R} \lim _{\varepsilon} w_{\varepsilon, R}$. From the second proof, $w(x, t)=\tilde{w}(y, t)$ and $\tilde{w}=\lim _{R} \lim _{\varepsilon} \tilde{w}_{\varepsilon, R}$. Both relations will be needed in $\$ 3.3$.

REMARK 2. From (3.12) and the maximum principle applied to $w=w_{\varepsilon, R}$ we deduce that

$$
\left|w_{\varepsilon, R}\right| \leqslant A
$$

where $A$ is a constant independent of $\varepsilon, R$ and of $s(t)$. Taking $\varepsilon \downarrow 0, R \uparrow \infty$ we deduce that

$$
0 \leqslant w(x, t) \leqslant A
$$


with the same $A$, which is independent of the curve $s(t)$.

REMARK 3. We can write, for $w=w_{\varepsilon, R}$,

$$
\left(w-\gamma_{1}\right)_{t}-\left(w-\gamma_{1}\right)_{x x}=-1-\beta_{\varepsilon}(w)-\gamma_{1 t}+\gamma_{1 x x} \equiv B
$$

where, by (3.11), $B$ is bounded by a constant $A_{0}$ independent of $\varepsilon, R$ and the curve $s(t)$. Multiplying (3.21) by $w-\gamma_{1}$ and integrating over $G_{R}$, we obtain, after using (3.19) and taking $\varepsilon \downarrow 0$,

$$
-\iint_{G_{R}}\left(w-\gamma_{1}\right)\left(w-\gamma_{1}\right)_{x x} d x d t \leqslant A_{1}
$$

where $w=w_{R}$ and $A_{1}$ is a constant independent of $R$ and of the curve $s(t)$. If $R$ is sufficiently large then $w=w_{x}=0$ on $x=-R$. Hence, integrating by parts we find, after letting $R \rightarrow \infty$, that

$$
\iint_{G} w_{x}^{2}(x, t) d x d t \leqslant A
$$

where $A$ is a constant independent of the curve $s(t)$.

3.3. Further properties of $w$. We shall need the conditions:

$$
\gamma_{1 x} \geqslant 0, \gamma_{1 t} \geqslant 0 \text { if } x \geqslant 1, \quad k_{1 x} \geqslant 0 \text { if }-1 \leqslant x \leqslant 1 .
$$

Lemma 3.2. The function $w_{x}(x, t)$ is continuously differentiable in $\bar{G}$ and $w_{x} \geqslant 0$.

Proof. The function $\tilde{w}=\tilde{w}_{\varepsilon, R}(y, t)$, occurring in the second proof of Lemma 3.1, satisfies

$$
\tilde{w}_{t}-\frac{y \dot{s}(t)}{s(t)} \tilde{w}_{y}-\frac{1}{s^{2}(t)} \tilde{w}_{y y}+\beta_{\varepsilon}(\tilde{w})=-1
$$

Differentiating this equation with respect to $t$, multiplying by $\left|\tilde{w}_{t}\right|^{p-2} \tilde{w}_{t}$ $(2 \leqslant p<\infty)$ and integrating over $\tilde{G}_{R}$, we find (cf. [15]) that

$$
\int_{-R}^{1}\left|\frac{\partial}{\partial t} \tilde{w}_{e, R}\right|^{p} d y<C \quad(0<t<T)
$$

where $C$ is a constant independent of $\varepsilon, R$. We can now apply the elliptic $L^{p}$ estimates to the elliptic operator $y \dot{s} \tilde{w}_{y} / s+\tilde{w}_{y y} / s^{2}$ and conclude that

$$
\int_{-R}^{1}\left[\left|\frac{\partial}{\partial y} \tilde{w}_{\varepsilon, R}\right|^{p}+\left|\frac{\partial^{2}}{\partial y^{2}} \tilde{w}_{\varepsilon, R}\right|^{p}\right] d y<C \quad(0<t<T)
$$

where $C$ is a constant independent of $\varepsilon, R$.

Taking $\varepsilon \downarrow 0, R \uparrow \infty$, we deduce from (3.25), (3.26), after performing the transformation (3.14), that 


$$
\int_{R^{l}}\left[\left|w_{t}(x, t)\right|^{p}+\left|w_{x}(x, t)\right|^{p}+\left|w_{x x}(x, t)\right|^{p}\right] d x<C \quad(0<t<T) .
$$

Since $p$ is arbitrary, the Sobolev inequality implies that $w$ is continuous in $\bar{G}$, and that the function $x \rightarrow w_{x}(x, t)$ is uniformly Hölder continuous in $x$, with exponent and coefficient that are independent of $t$. By the proof of Corollary 2.7 of [19] we then deduce that $w_{x}$ is continuous in $\bar{G}$.

Set

$$
\Omega=\{(x, t) \in G ; w(x, t)>0\}, \quad \Omega_{\tau}=\Omega \cap\{t<\tau\} .
$$

The conditions on $\gamma_{1}$ in (3.23) together with the fact that $s(t)$ is increasing in $t$ imply that

$$
w(s(t), t)=\gamma_{1}(s(t), t) \text { is increasing in } t .
$$

Since $k_{1 x} \geqslant 0, k_{1}(1)=\gamma_{1}(1,0)$, we also have that

$$
w(s(t), t) \geqslant k_{1}(x) \text { if } x<1
$$

We now apply the maximum principle to the function $w$ in $\Omega_{\tau}$. Since $w_{t}-w_{x x}=-1<0$ in $\Omega$ and since $w=0$ in that part of the parabolic boundary of $\Omega$ that lies in $G$, we deduce (using (3.28), (3.29)) that the maximum of $w$ in $\bar{\Omega}_{\tau}$ is attained at $(s(\tau), \tau)$. Consequently,

$$
w_{x}(s(\tau), \tau) \geqslant 0 \quad \text { if } 0<\tau<t .
$$

Next,

$$
w_{x}(x, 0)=k_{1 x} \geqslant 0 \text { if }-1<x<1 .
$$

Finally, $w_{x}=0$ on the free boundary. Since $w_{x}$ is continuous in $\bar{\Omega}$ and

$$
\left(w_{x}\right)_{t}-\left(w_{x}\right)_{x x}=0 \text { in } \Omega
$$

the maximum principle applied to $w_{x}$ in $\Omega$ yields $w_{x}>0$ in $\Omega$. Since $w_{x}=0$ in $G \backslash \Omega$, the proof of the lemma is complete.

We shall need the following additional assumptions:

$$
k_{1 x x}(x)-1 \geqslant 0 \text { if }-1<x<1
$$

there is a function $\tilde{\gamma}_{1}(x, t)$ with continuous derivatives $\tilde{\gamma}_{1 x}, \tilde{\gamma}_{1 t}, \tilde{\gamma}_{1 x x}$ such that

$$
\begin{aligned}
& \tilde{\gamma}_{1}(x, t)=\gamma_{1}(x, t) \text { if } x>1, \\
& \tilde{\gamma}_{1}(x, 0) \leqslant k_{1}(x) \text { if } x<1, \\
& \lim _{x \rightarrow-\infty} \tilde{\gamma}_{1}(x, t) \leqslant 0 \text { uniformly in } t, 0 \leqslant t \leqslant T, \\
& \tilde{\gamma}_{1 x x}-\tilde{\gamma}_{t} \geqslant 1 \text { if } x \in R^{1}, 0<t<T .
\end{aligned}
$$

For example, we can take $\tilde{\gamma}_{1}(x, t)=k_{1}(x)+(x-1) t$ if $\gamma_{1}(x, t)=k_{1}(x)+$ $(x-1) t$ when $x>1$, provided $k_{1 x x}(x) \geqslant x$ whenever $x>1$.

LEMMA 3.3. Under the additional assumptions (3.30), (3.31), 


$$
\begin{gathered}
w(x, t)>\gamma_{1}(x, t) \quad \text { if } 1<x<s(t), 0<t<T, \\
w_{x}-\gamma_{1 x} \leqslant 0 \quad \text { if } x=s(t), 0<t<T .
\end{gathered}
$$

Proof. Consider the function

$$
u(x, t)=w_{\varepsilon, R}(x, t)-\tilde{\gamma}_{1}(x, t)
$$

in the region $G_{R}$. From the last relation in (3.31) it follows that

$$
u_{t}-u_{x x} \geqslant-\beta_{\varepsilon}\left(w_{\varepsilon, R}\right) \geqslant 0 \text { in } G_{R} .
$$

Also, for any $\delta>0$,

$$
\begin{aligned}
& u=0 \quad \text { if } x=s(t), 0<t<T, \\
& u(x, 0) \geqslant 0 \quad \text { if }-R<x<1, \\
& u(-R, t) \geqslant-\delta \quad \text { if } 0<t<T,
\end{aligned}
$$

provided $R$ is sufficiently large (depending on $\delta$, but not on $\varepsilon$ ). By the maximum principle, $u \geqslant-\delta$ in $G_{R}$. Taking $\varepsilon \rightarrow 0, R \rightarrow \infty$ we get

$$
w-\tilde{\gamma}_{1} \geqslant-\delta \text { in } G \text {. }
$$

Since $\delta$ is arbitrary, $w \geqslant \tilde{\gamma}_{1}$ in $G$. By the strong maximum principle we actually then have $w>\tilde{\gamma}_{1}$ in $\Omega$. This gives (3.32). The inequality (3.33) is an immediate consequence of (3.32).

LEMMA 3.4. Under the additional assumptions (3.30), (3.31), $w_{t} \geqslant 0$ a.e.

Proof. Consider first the case where $\gamma_{1}, k_{1}, s$ are in $C^{3}$ and

$$
\gamma_{1 x}(1,0) \dot{s}(0)+\gamma_{1 t}(1,0)-k_{1 x x}(1)=0 .
$$

This is a consistency condition for the equation

$$
\frac{\partial}{\partial t} w_{\varepsilon, R}-\frac{\partial^{2}}{\partial x^{2}} w_{\varepsilon, R}+\beta_{\varepsilon}\left(w_{\varepsilon, R}\right)=-1
$$

at the point $(1,0)$, since $\beta_{\varepsilon}(0)=-1$. At $(-R, 0)$ the consistency condition for (3.35) is also satisfied. We can now apply the Schauder estimates [13] to deduce that $\partial w_{\varepsilon, R} / \partial x, \partial^{2} w_{\varepsilon, R} / \partial x^{2}, \partial w_{\varepsilon, R} / \partial t$ are continuous in $\bar{G}_{R}$.

Differentiating the relation

$$
w_{\varepsilon, R}(s(t), t)-\gamma_{1}(s(t), t)=0,
$$

we get

$$
\dot{s}(t) \frac{\partial}{\partial x}\left(w_{\varepsilon, R}-\gamma_{1}\right)(s(t), t)+\frac{\partial}{\partial t} w_{\varepsilon, R}(s(t), t)=\gamma_{1 t}(s(t), t) .
$$

Since $w_{\varepsilon, R} \rightarrow w$ (weakly in $L^{p}\left(G_{R}\right)$ together with the first derivatives and, therefore, uniformly in $\bar{G}_{R}$ ), we can apply the Schauder boundary estimates (the consistency condition at $(1,0)$ is used here) to deduce that 


$$
\frac{\partial}{\partial x} w_{\varepsilon, R}(s(t), t) \rightarrow \frac{\partial}{\partial x} w(s(t), t) \quad \text { as } \varepsilon \rightarrow 0, R \rightarrow 0,
$$

uniformly in $t, 0 \leqslant t \leqslant T$.

Using Lemma 3.3 we find that

$$
\frac{\partial}{\partial x}\left(w_{\varepsilon, R}-\gamma_{1}\right)(s(t), t)<\frac{\delta}{A}
$$

if $0<\varepsilon<\varepsilon_{0}, R>R_{0}$ for some $\varepsilon_{0}, R_{0}$ depending on $\delta / A$; here $\delta$ is an

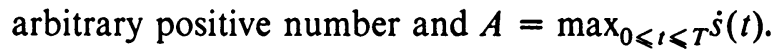

Using this inequality in (3.36), and recalling that $\gamma_{1 t} \geqslant 0$, we get

$$
\frac{\partial}{\partial t} w_{\varepsilon, R}(s(t), t)>-\delta \quad\left(0<\varepsilon<\varepsilon_{0}, R>R_{0}\right) .
$$

Setting $\xi=\partial w_{\varepsilon, R} / \partial t$ and differentiating (3.35) with respect to $t$, we get

$$
\xi_{t}-\xi_{x x}+\beta^{\prime}\left(w_{\varepsilon, R}\right) \xi=0 \text { in } G_{R} .
$$

Since $\beta_{\varepsilon}(0)=-1$ and since (3.30) holds,

$$
\xi(x, 0)=-1-\beta_{\varepsilon}\left(k_{1}(x)\right)+k_{1 x x}(x) \geqslant 0 \text { if }-R<x<1 .
$$

On $x=-R, \xi=0$. On $x=s(t), \xi>-\delta$, by (3.37). Since $\xi$ is continuous in $\bar{G}_{R}$, we can apply the maximum principle to conclude that $\xi \geqslant-\delta$ in $\bar{G}_{R}$. Taking $\varepsilon \rightarrow 0, R \rightarrow \infty$ the assertion of the lemma follows.

We have assumed in the above proof that $\gamma_{1}, k_{1}, s$ are in $C^{3}$ and (3.34) holds. In the general case, we approximate $\gamma_{1}, k_{1}, s$ by $C^{3}$ functions $\gamma_{1}^{m}, k_{1}^{m}$, $s^{m}$ satisfying all the assumptions of Lemma 3.4 and (3.34). For the corresponding variational inequality, the solution $w^{m}$ satisfies $\partial w^{m} / \partial t \geqslant 0$. By uniqueness, $w^{m} \rightarrow w$ (uniformly, say) as $m \rightarrow \infty$, and the assertion of the lemma readily follows.

We now introduce the free boundary curve $x=\sigma(t)$ of the variational inequality (3.9):

$$
\sigma(t)=\inf \{x ; x<s(t), w(x, t)>0\}, \quad 0 \leqslant t \leqslant T .
$$

Lemma 3.5. Let $s(t)$ be a monotone increasing function with Hölder continuous derivative, for $0 \leqslant t \leqslant T$, such that $s(0)=1$. Let $\gamma_{1}, k_{1}$ satisfy the conditions in (3.4), (3.23), (3.30), (3.31). Then there exists a unique solution $w$ with compact support of (3.9); $w$ and $w_{x}$ are continuous in $\bar{G}, w_{x} \geqslant 0, w_{t} \geqslant 0$ a.e. in G. Furthermore, the function $\sigma(t)$ is continuous and monotone decreasing in $t, 0 \leqslant t \leqslant T$.

Proof. All the assertions of the lemma, except those regarding $\sigma(t)$, follow from Lemmas 3.1, 3.2, 3.4. Since $w_{x} \geqslant 0, w_{t} \geqslant 0$ a.e. in $G$, the strong maximum principle implies that $w_{x}>0, w_{t}>0$ in $\Omega$. It follows that $(x$, $t) \in \Omega$ if and only if $\sigma(t)<x<s(t)$ and $\sigma(t)$ is monotone decreasing. 
Clearly $\sigma(t)$ is upper semicontinuous function. Hence $\sigma(t)$ is right continuous. It remains to prove that $\sigma(t)$ is left continuous. If this is not the case then there is a point $t_{0} \in[0, T)$ such that $\sigma\left(t_{0}+0\right)<\sigma\left(t_{0}\right)$. Introduce the line segment $I=\left\{\left(x, t_{0}\right) ; \sigma\left(t_{0}+0\right)<x<\sigma\left(t_{0}\right)\right\}$. Then $I$ is a part of the boundary of $\Omega$. Since $D=\left\{(x, t) ; \sigma\left(t_{0}+0\right)<x<\sigma\left(t_{0}\right), t_{0}<t<T\right\}$ is contained in $\Omega, w_{t}-w_{x x}=-1$ in $D, w=0$ on $I$; it follows that $w$ is smooth in $D \cup I$. But then $w_{t}=-1+w_{x x}=-1<0$ on I. It follows that $w(x, t)<0$ at the points $(x, t)$ of $\Omega$ such that $\sigma\left(t_{0}+0\right)<x<\sigma\left(t_{0}\right)$ and such that $t>t_{0}, t-t_{0}$ is sufficiently small. This is impossible since $w>0$ in $\Omega$.

\subsection{The case where $s(t)$ is not smooth.}

LEMMA 3.6. Let the conditions of Lemma 3.5 hold and let $s^{\prime}(t)$ be a monotone increasing function with Hölder continuous derivative for $0 \leqslant t \leqslant T$ such that $s^{\prime}(0)=1$. Denote by $w^{\prime}(x, t)$ the solution of the variational inequality (3.9) with $s(t)$ replaced by $s^{\prime}(t)$, and denote by $x=\sigma^{\prime}(t)$ the corresponding free boundary. If $s^{\prime}(t) \geqslant s(t)$ for $0 \leqslant t \leqslant T$, then

$$
\begin{gathered}
w^{\prime}(x, t) \geqslant w(x, t) \text { for all } 0 \leqslant x \leqslant s(t), 0 \leqslant t \leqslant T, \\
\sigma^{\prime}(t) \leqslant \sigma(t) \text { for all } 0 \leqslant t \leqslant T .
\end{gathered}
$$

Proof. We compare the solution $w_{\varepsilon, R}$ of (3.10) with the corresponding solution $w_{\varepsilon, R}^{\prime}$ when $s$ is replaced by $s^{\prime}$. On $x=-R$ and on $t=0, w_{\varepsilon, R}^{\prime}-w_{\varepsilon, R}$ $=0$. Next, by the proof of Lemma 3.3, for any $\delta>0, w_{\varepsilon, R}^{\prime}>\gamma_{1}-\delta$ on $x=s(t)$ provided $R$ is sufficiently large, depending only on $\delta$. Since $w_{\varepsilon, R}=\gamma_{1}$ on $x=s(t)$, we have $w_{\varepsilon, R}^{\prime}-w_{\varepsilon, R}>-\delta$ on $x=s(t)$. Applying the maximum principle to $w_{\varepsilon, R}^{\prime}-w_{\varepsilon, R}$, we find that $w_{\varepsilon, R}^{\prime}-w_{\varepsilon, R}>-\delta$ in $G_{R}$. Taking $\varepsilon \rightarrow 0$, $R \rightarrow \infty$ we conclude that $w^{\prime}-w \geqslant-\delta$ in $G$. Since $\delta$ is arbitrary the assertion (3.39) follows. Clearly, (3.40) is a consequence of (3.39).

We shall now consider the variational inequality (3.9) in case $s(t)$ is any monotone increasing function with $s(0)=1$. We shall construct a "generalized" solution as a limit of solutions $w_{n}$ of variational inequalities corresponding to smooth curves $s_{n}(t)$. In order to define $s_{n}(t)$, we define $s(t)=1$ if $t<0$,

$$
\begin{aligned}
& \rho(t)= \begin{cases}c \exp [1 /(|t-1|-1)] & \text { if }|t-1|<1, \\
0 & \text { if }|t-1| \geqslant 1,\end{cases} \\
& \rho_{n}(t)=n \rho(n t) \quad \text { if } n=1,2, \ldots,
\end{aligned}
$$

where $c$ is a positive constant such that $\int_{R^{\prime}} \rho(t) d t=1$.

Let

$$
s_{n}(t)=\int_{R^{1}} \rho_{n}(t-\tau) s(\tau) d \tau=\int_{t-2 / n}^{t} \rho_{n}(t-\tau) s(\tau) d \tau .
$$


We can write

$$
s_{n}(t)=\int_{R^{1}} \rho_{n}(\tau) s(t-\tau) d \tau=\int_{0}^{2} \rho(\tau) s(t-\tau / n) d \tau
$$

From (3.41) we see that $s_{n}(t)$ is a $C^{\infty}$ function, and from (3.42) we see that $s_{n}(t)$ is a monotone increasing function in $t, s_{n}(0)=1$, and

$$
s_{n}(t) \leqslant s_{n+1}(t) \text {. }
$$

Denote by $w_{n}$ the solution of the variational inequality (3.9) corresponding to $s=s_{n}$ and denote by $x=\sigma_{n}(t)$ the corresponding free boundary curve. By Lemma 3.6,

$$
\begin{gathered}
w_{n} \leqslant w_{n+1} \text { if }-\infty<x<s_{n}(t), 0 \leqslant t \leqslant T, \\
\sigma_{n+1}(t)<\sigma_{n}(t) \text { if } 0 \leqslant t<T .
\end{gathered}
$$

Set

$$
\begin{gathered}
w(x, t)=\lim _{n \rightarrow \infty} w_{n}(x, t) \quad \text { if }-\infty<x<s(t), 0<t<T, \\
\sigma(t)=\lim _{n \rightarrow \infty} \sigma_{n}(t) \quad \text { if } 0 \leqslant t \leqslant T .
\end{gathered}
$$

Let $s^{*}(t)$ be any $C^{\infty}$ function such that $s^{*}(t) \leqslant s(t)-\delta$ if $0 \leqslant t \leqslant T$, for some $\delta>0$, and such that $\tilde{\gamma}_{1}(x, t)>0$ in a $\bar{G}$-neighborhood of

$$
\Gamma^{*}=\left\{\left(s^{*}(t), t\right) ; 0 \leqslant t<T\right\} .
$$

By Remark 2 at the end of $\$ 3.2,\left|w_{n}\right| \leqslant A$ in some $\bar{G}$-neighborhood $N$ of $\Gamma^{*}$, where $A$ and $N$ are both independent of $n$. By the proof of Lemma 3.3, $w_{n} \geqslant \tilde{\gamma}_{1}>0$ in $N$ and, consequently, $w_{n t}-w_{n x x}=-1$ in $N$. By standard results on parabolic equations we then deduce (if $k_{1 x x}$ is Hölder continuous) that $w_{n x}, w_{n t}, w_{n x x}$ are continuous and uniformly bounded in some smaller $\bar{G}$-neighborhood of $\Gamma^{*}$.

We can now consider $w_{n}$ as a solution of the variational inequality (3.9) with $s(t)$ replaced by $s^{*}(t)$. From the proof of Lemma 3.1 we get, for any $1<p<\infty$,

$$
\int_{-\infty}^{s^{*}(t)}\left[\left|w_{n x}\right|^{p}+\left|w_{n t}\right|^{p}+\left|w_{n x x}\right|^{p}\right] d x \leqslant C \text { if } 0<t<T
$$

where $C$ is a constant independent of $n$; by approximation, this inequality holds also when $k_{1 x x}$ is not assumed to be Hölder continuous.

We can now use the Sobolev inequality to deduce that

$$
\begin{aligned}
& w_{n} \text { is uniformly Hölder continuous in }(x, t), \\
& \text { uniformly with respect to } n, \\
& w_{n x} \text { is uniformly Hölder continuous in } x, \\
& \text { uniformly with respect to } t, n \text {. }
\end{aligned}
$$

In view of (3.46) we then have 


$$
w_{n} \rightarrow w \text { uniformly in } \bar{G}^{*},
$$

$w_{x}$ is Hölder continuous in $x$, uniformly in $t$, where

$$
G^{*}=\left\{(x, t) ;-\infty<x<s^{*}(t), 0<t<T\right\} .
$$

Notice also that

$$
\int_{-\infty}^{s^{*}(t)}\left[\left|w_{x}\right|^{p}+\left|w_{t}\right|^{p}+\left|w_{x x}\right|^{p}\right] d x \leqslant C \quad \text { if } 0<t<T .
$$

From (3.52) and the proof of Corollary 2.7 in [19] we also deduce that

$$
w_{x} \text { is continuous in } \bar{G}^{*} \text {. }
$$

Definition. We shall call the function $w(x, t)$ a generalized solution of the variational inequality (3.9). The curve $x=\sigma(t)$ will be called the free boundary.

Notice that if $w$ were continuous up to the curve $x=s(t)$ and if $w_{x}, w_{t}, w_{x x}$ were in $L^{2}$ in a $G$-neighborhood of this curve, then $w$ would be a solution of the variational inequality (3.9) in the usual sense.

LEMMA 3.7. Let $s(t)$ be a monotone increasing function for $0 \leqslant t \leqslant T$, with $s(0)=1$, and let $\gamma_{1}, k_{1}$ satisfy the conditions in (3.4), (3.23), (3.30), (3.31). Then

$$
\sigma(t)=\inf \{x ; w(x, t)>0\}
$$

$w(x, t)=0$ if $x<\sigma(t)$, and the function $\sigma(t)$ is monotone decreasing and continuous for $0 \leqslant t \leqslant T$.

PRoof. Let

$$
\begin{aligned}
\Omega & =\{(x, t) \in G ; w(x, t)>0\}, \\
\Omega_{n} & =\left\{(x, t) \in G ; w_{n}(x, t)>0\right\}, \\
\Omega_{0} & =\{(x, t) \in G ; x>\sigma(t)\} .
\end{aligned}
$$

In view of (3.44), (3.46), $\Omega_{n} \subset \Omega$. Hence

$$
\Omega_{0}=\bigcup_{n=1}^{\infty} \Omega_{n} \subset \Omega
$$

On the other hand, $w_{n}\left(\sigma_{n}(t), t\right)=0$. Taking $n \rightarrow \infty$ we get $w(\sigma(t), t)=0$. Observing that $w_{x} \geqslant 0$ a.e. (since $w_{n x} \geqslant 0$ a.e.) we deduce that $w(x, t)=0$ if $x<\sigma(t)$. This, together with (3.56), completes the proof of (3.55).

It is clear that $\sigma(t)$ is monotone decreasing in $t$. The proof that $\sigma(t)$ is continuous is the same as in the proof of Lemma 3.5.

Remark. On $x=s(t)$ the generalized solution $w$ may not be continuous. From Remark 3 at the end of $\S 3.2$, when applied to $w_{n}$ with $n \rightarrow \infty$, we see that

$$
\int_{0}^{T} \int_{-\infty}^{s(t)} w_{x}^{2} d x d t<\infty
$$


We conclude this section with a comparison lemma.

LEMMA 3.8. Let the condition of Lemma 3.7 hold and let $s^{\prime}(t)$ be a monotone increasing function for $0 \leqslant t \leqslant T$, with $s^{\prime}(0)=1$. Denote by $w^{\prime}(x, t)$ the generalized solution of (3.9) corresponding to $s^{\prime}$, and denote its free boundary curve by $x=\sigma^{\prime}(t)$. If $s^{\prime}(t) \geqslant s(t)$ for $0 \leqslant t \leqslant T$, then

$$
\begin{aligned}
w^{\prime}(x, t) & \geqslant w(x, t) \quad \text { if }-\infty<x<s(t), 0 \leqslant t \leqslant T, \\
\sigma^{\prime}(t) & \leqslant \sigma(t) \quad \text { if } 0 \leqslant t \leqslant T .
\end{aligned}
$$

Proof. Denote by $s_{n}^{\prime}$ the functions defined by (3.41) when $s$ is replaced by $s^{\prime}$. Then $s_{n}^{\prime}(t) \geqslant s_{n}(t)$. Now apply Lemma 3.6 to the pair $s_{n}(t), s_{n}^{\prime}(t)$ and take $n \rightarrow \infty$.

3.5. Existence of solutions for the Q.V.I. Let $\sigma(t)$ be a monotone decreasing function for $0 \leqslant t \leqslant T$, with $\sigma(0)=-1$. Consider the variational inequality: Find $\bar{w}(x, t)$ such that

$$
\begin{aligned}
& \bar{w} \geqslant 0 \quad \text { if } x>\sigma(t), 0<t<T, \\
& \bar{w}_{t}, \bar{w}_{x}, \bar{w}_{x x} \text { are in } L^{2}(\hat{G}) \quad \text { where } \hat{G}=\{(x, t) ; \sigma(t)<x<\infty, 0<t<T\}, \\
& \int_{\sigma(t)}^{\infty}\left(\bar{w}_{t}-\bar{w}_{x x}\right)(v-\bar{w}) d x \geqslant-\int_{\sigma(t)}^{\infty}(v-\bar{w}) d x \quad \text { for a.a. } t \in(0, T), \\
& \quad \text { for any } v=v(x) \in L^{\infty}\left(R^{1}\right), v \geqslant 0 \text { a.e., } \\
& \bar{w}=\gamma_{2} \text { if } x=\sigma(t), 0<t<T, \\
& \bar{w}(x, 0)=k_{2}(x) \quad \text { if } x>-1 .
\end{aligned}
$$

We shall assume that

$$
\gamma_{2 x} \leqslant 0, \gamma_{2 t} \geqslant 0 \text { if } x \leqslant-1, \quad k_{2 x} \leqslant 0 \text { if }-1 \leqslant x \leqslant 1 .
$$

Then we can prove an analog of Lemma 3.2, namely, if $\sigma(t)$ has Hölder continuous first derivatives then $w_{x} \leqslant 0$.

Next we assume

$$
k_{2 x x}(x)-1 \geqslant 0 \text { if }-1<x<1 ;
$$

there is a function $\tilde{\gamma}_{2}(x, t)$ with continuous derivatives $\tilde{\gamma}_{2 x}, \tilde{\gamma}_{2 t}, \tilde{\gamma}_{2 x x}$ such that

$$
\begin{aligned}
& \tilde{\gamma}_{2}(x, t)=\gamma_{2}(x, t) \text { if } x<-1, \\
& \tilde{\gamma}_{2}(x, 0) \leqslant k_{2}(x, 0) \text { if } x>-1, \\
& \varlimsup_{x \rightarrow \infty} \tilde{\gamma}_{2}(x, t) \leqslant 0 \text { uniformly in } t, 0 \leqslant t \leqslant T, \\
& \tilde{\gamma}_{2 x x}-\tilde{\gamma}_{t} \geqslant 1 \text { if } x \in R^{1}, 0<t<T .
\end{aligned}
$$


Then we can establish, analogously to Lemmas 3.3, 3.4, that

$$
\begin{gathered}
\bar{w}(x, t)>\gamma_{2}(x, t) \quad \text { if } \sigma(t)<x<-1,0<t<T, \\
\bar{w}_{x}-\gamma_{2 x} \geqslant 0 \quad \text { if } x=\sigma(t), 0<t<T, \\
\bar{w}_{t} \geqslant 0 \text { a.e. }
\end{gathered}
$$

Next one can establish an analog of Lemma 3.5 for the variational inequality (3.57).

Consider now the case where $\sigma(t)$ is merely assumed to be monotone decreasing with $\sigma(0)=-1$. Define $\sigma(t)=-1$ if $t<0$, and

$$
\sigma_{n}(t)=\int_{R^{1}} \rho_{n}(t-\tau) \sigma(\tau) d \tau .
$$

Denote by $\bar{w}_{n}$ the solution of (3.57) when $\sigma(t)$ is replaced by $\sigma_{n}(t)$, and denote by $x=\bar{s}_{n}(t)$ the corresponding free boundary. Since $\sigma_{n+1}(t)<\sigma_{n}(t)$, we have, by an analog of Lemma 3.6,

$$
\bar{w}_{n} \leqslant \bar{w}_{n+1}, \quad \bar{s}_{n} \leqslant \bar{s}_{n+1} .
$$

Let

$$
\bar{w}(x, t)=\lim _{n \rightarrow \infty} \bar{w}_{n}(x, t), \quad \bar{s}(t)=\lim _{n \rightarrow \infty} \bar{s}_{n}(t) .
$$

We call $\bar{w}$ the generalized solution of the variational inequality (3.57), and we call $x=\bar{s}(t)$ the free boundary curve for (3.57).

An analog of Lemma 3.7 is valid. In particular,

$$
\begin{aligned}
& \bar{w}(x, t)>0 \text { if } \sigma(t)<x<\bar{s}(t), \\
& \bar{w}(x, t)=0 \text { if } x>\bar{s}(t), \\
& \bar{s}(t) \text { is monotone increasing and continuous for } 0 \leqslant t \leqslant T .
\end{aligned}
$$

Definition of $W$. Let $\Sigma$ denote the class of all monotone increasing functions $s(t), 0 \leqslant t \leqslant T$, with $s(0)=1$. For any $s \in \Sigma$, denote by $x=\sigma(t)$ the free boundary of the generalized solution of the variational inequality (3.9). Denote by $x=\bar{s}(t)$ the free boundary of the generalized solution of the variational inequality (3.57). Then the mapping $s \rightarrow \bar{s}$ is denoted by $W$, i.e., $\bar{s}=W s$.

Notice that every $s \in \Sigma$ determines a generalized solution $w$ of (3.9) and a free boundary $x=\sigma(t)$ which, in turn, determines a generalized solution $\bar{w}$ of (3.57).

Definition. If for some $s \in \Sigma, W s=s$ then the corresponding pair $(w, \bar{w})$ is said to form a generalized solution of the Q.V.I. (3.6), (3.7). The curves $x=s(t), x=\sigma(t)$ are called the free boundary curves.

In view of Lemma 3.7 (and the analogous result for (3.57)), the free boundary curves are continuous.

The set $\Sigma$ is partially ordered by the relation: 


$$
s_{1} \prec s_{2} \text { if and only if } s_{1}(t) \leqslant s_{2}(t) \text { for } 0 \leqslant t \leqslant T \text {. }
$$

Notice that every subset of elements $s_{\alpha}$ in $\Sigma$ has an upper bound $s$ in $\Sigma$, namely, $s(t)=\sup _{\alpha} s_{\alpha}(t)$, and a lower bound $\tilde{s}$ in $\Sigma$, namely, $\tilde{s}(t)=\inf _{\alpha} s_{\alpha}(t)$.

By Lemma 3.8 and its counterpart for the variational inequality (3.57) we see that if $s_{1} \prec s_{2}$ then $W s_{1} \prec W s_{2}$, i.e., $W$ is a monotone increasing mapping.

Now, from the methods of [10] it follows that the support of the solution $\bar{w}$ of (3.57) (for any smooth function $\sigma$ ) is bounded uniformly with respect to $\sigma$. Consequently there is a constant $H$ such that $\bar{s}(t) \leqslant H$ whenever $\bar{s}=W s$, $s \in \Sigma$. Defining

$$
s_{1}(t)= \begin{cases}0 & \text { if } t=0, \\ H & \text { if } 0<t \leqslant T,\end{cases}
$$

we conclude that $s_{1}>W s_{1}$.

If $s_{2}(t) \equiv 1$ then clearly $s_{2} \prec W s_{2}$.

Thus, $W$ satisfies the conditions in Lemma 2 of Tartar [23]. We deduce from this lemma that $W$ has a maximal fixed point $s^{*}$ and a minimal fixed point $s_{*}$ in the interval $\left(s_{1}, s_{2}\right)$. Since every fixed point must lie in this interval, we conclude that

$$
s^{*} \succ s_{*}, W s^{*}=s^{*}, W s_{*}=s_{*}, \quad \text { if } W s=s \text { then } s^{*} \succ s \succ s_{*} .
$$

Denote by $\left(w^{*}, \bar{w}^{*}\right)$ the generalized solution of (3.6), (3.7) corresponding to $s^{*}$, and denote by $\left(w_{*}, \bar{w}_{*}\right)$ the generalized solution of (3.6), (3.7) corresponding to $s_{*}$. We then have:

THEOREM 3.1. Let the conditions (3.4), (3.23), (3.30), (3.31) and (3.58)-(3.60) hold. Then:

(i) There exist generalized solutions $\left(w^{*}, \bar{w}^{*}\right),\left(w_{*}, \bar{w}_{*}\right)$ of (3.6), (3.7) with the corresponding free boundary functions $\left(s^{*}, \sigma^{*}\right)$ and $\left(s_{*}, \sigma_{*}\right)$.

(ii) If $(w, \bar{w})$ is any generalized solution of (3.6), (3.7) with the corresponding free boundary functions $(s, \sigma)$, then

$$
\begin{aligned}
& s_{*} \leqslant s \leqslant s^{*}, \quad \sigma^{*} \leqslant \sigma \leqslant \sigma_{*}, \\
& w_{*} \leqslant w \leqslant w^{*}, \quad \bar{w}^{*} \leqslant \bar{w} \leqslant \bar{w}_{*},
\end{aligned}
$$

each inequality in (3.63) is valid in the set where both sides are defined.

(iii) For any solution $(w, \bar{w})$ the free boundary functions $s(t), \sigma(t)$ are continuous functions for $0 \leqslant t \leqslant T ; s(t)$ is monotone increasing and $\sigma(t)$ is monotone decreasing.

\section{Chapter 4. Stefan Type Free Boundary Problem for Systems}

In Chapters 2, 3 we have solved Q.V.I. by a fixed point theorem for a monotone increasing operator. In Chapter 5 we shall use a method of integral 
equations in order to find a unique regular solution with regular free boundaries of the parabolic Q.V.I. of Chapter 3 in the special case of zero-sum game. This method of integral equations is based on the fact that the Q.V.I. (for zero-sum game) can be reformulated as a Stefan problem of melting of ice; for the case of one player, this fact was first noted by Van Moerbeke [24].

In this chapter we study (by the method of integral equations) a Stefan type free boundary problem for a system with two "temperatures," $\theta_{1}$ and $\theta_{2}$. The system in Chapter 5 is somewhat different (and, in some sense, it is a special case); it can be studied by the same methods as in this chapter.

4.1. Existence and uniqueness; the increasing case. Let $g_{1}(x), g_{2}(x)$ be functions defined for $-1 \leqslant x \leqslant 1$ and let $\lambda_{1}(x, t), \lambda_{2}(x, t)$ be functions defined for $-\infty<x<\infty, t \geqslant 0$, satisfying:

$$
\begin{aligned}
& g_{i}(x) \text { are continuously differentiable and } \geqslant 0 \text { for }-1 \leqslant x \leqslant 1 ; \\
& \left|g_{i x}\right| \leqslant C ; g_{1}(-1)=0, g_{2}(1)=0 ; \\
& \lambda_{i}(x, t) \text { are continuously differentiable and } \geqslant 0 ; \\
& \left|\lambda_{i x}\right|,\left|\lambda_{i t}\right| \leqslant C ; \lambda_{1}(1,0)=g_{1}(1), \lambda_{2}(-1,0)=g_{2}(-1) .
\end{aligned}
$$

We consider the following problem: find functions $\theta_{1}(x, t), \theta_{2}(x, t), s_{1}(t), s_{2}(t)$ such that

$$
\begin{aligned}
& s_{1}(t)<s_{2}(t), \quad s_{1}(0)=-1, \quad s_{2}(0)=1, \\
& \frac{\partial \theta_{i}}{\partial t}=\frac{\partial^{2} \theta_{i}}{\partial x^{2}} \quad \text { if } s_{1}(t)<x<s_{2}(t), t>0 \\
& \theta_{i}\left(s_{i}(t), t\right)=0 \quad \text { if } t \geqslant 0 \\
& \theta_{1}=\lambda_{1} \quad \text { if } x \geqslant s_{2}(t), t \geqslant 0, \\
& \theta_{2}=\lambda_{2} \text { if } x \leqslant s_{1}(t), t \geqslant 0, \\
& \left.\frac{\partial}{\partial x} \theta_{i}(x, t)\right|_{x=s_{i}(t)}=-\dot{s}_{i}(t) \quad \text { if } t \geqslant 0 \\
& \theta_{i}(x, 0)=g_{i}(x) \quad \text { if }-1 \leqslant x \leqslant 1 ; \\
& s_{i}(t) \text { is continuously differentiable for } t \geqslant 0, \\
& \partial \theta_{1} / \partial x \text { is continuous for } t \geqslant 0, x \leqslant s_{2}(t), \\
& \partial \theta_{2} / \partial x \text { is continuous for } t \geqslant 0, x \geqslant s_{1}(t), \\
& \partial \theta_{i} / \partial t, \partial^{2} \theta_{i} / \partial x^{2} \text { are continuous for } t>0, x \in\left(s_{1}(t), s_{2}(t)\right) .
\end{aligned}
$$

The main result of this section is the following

THEOREM 4.1. Under the assumptions (4.1), (4.2) there exists a unique solution $\left(\theta_{1}, \theta_{2}, s_{1}, s_{2}\right)$ of (4.3)-(4.6); furthermore $s_{1}(t)$ decreases and $s_{2}(t)$ increases as $t$ increases. 
Since $(-1)^{i} s_{i}(t)$ is increasing, we are dealing here with (what we call) the increasing case. Later on we shall prove a more general theorem whereby $(-1)^{i_{i}}(t)$ is not necessarily increasing.

The proof of Theorem 4.1 is based on the method by Friedman [12], [13, Chapter 8]. Some details will be omitted.

LEMMA 4.1. Let $s_{1}(t), s_{2}(t)$ be two curves satisfying, for $0 \leqslant t \leqslant \sigma$,

$$
\begin{aligned}
& s_{1}(t)<s_{2}(t), s_{i}(t) \text { is continuously differentiable, } \\
& s_{1}(0)=-1, s_{2}(0)=+1 .
\end{aligned}
$$

Let $u(x, t)$ be a solution of

$$
\frac{\partial u}{\partial t}=\frac{\partial^{2} u}{\partial x^{2}} \quad \text { if } x \in\left(s_{1}(t), s_{2}(t)\right), 0<t<\sigma,
$$

and let $\partial u / \partial x$ be continuous for $x \in\left[s_{1}(t), s_{2}(t)\right], 0 \leqslant t \leqslant \sigma$. Then we have, for $x \in\left(s_{1}(t), s_{2}(t)\right), 0<t \leqslant \sigma$, the integral representation of $u$,

$$
\begin{aligned}
u(x, t)= & \int_{-1}^{+1} K(x, t ; \xi, 0) u(\xi, 0) d \xi \\
& +\int_{0}^{t} \dot{s}_{2}(\tau) K\left(x, t ; s_{2}(\tau), \tau\right) u\left(s_{2}(\tau), \tau\right) d \tau \\
& -\int_{0}^{t} \dot{s}_{1}(\tau) K\left(x, t ; s_{1}(\tau), \tau\right) u\left(s_{1}(\tau), \tau\right) d \tau \\
& +\int_{0}^{t}\left[K(x, t ; \xi, \tau) \frac{\partial u}{\partial \xi}(\xi, \tau)-u(\xi, \tau) \frac{\partial K}{\partial \xi}(x, t ; \xi, \tau)\right]_{s_{1}(\tau)}^{s_{2}(\tau)} d \tau
\end{aligned}
$$

where

$$
K(x, t ; \xi, \tau)=\frac{1}{2 \pi^{1 / 2}(t-\tau)^{1 / 2}}=\exp \left[-\frac{(x-\xi)^{2}}{4(t-\tau)}\right] .
$$

The proof follows by using Green's formula with $u, K$ (see [12], [13]).

Suppose now that there is a solution of the problem (4.3)-(4.6). Let

$$
\begin{aligned}
& v_{1}(t)=\dot{s}(t)=-\frac{\partial \theta_{1}}{\partial x}\left(s_{1}(t), t\right), \\
& v_{2}(t)=-\dot{s}_{2}(t)=\frac{\partial \theta_{2}}{\partial x}\left(s_{2}(t), t\right), \\
& w_{1}(t)=-\frac{\partial \theta_{1}}{\partial x}\left(s_{2}(t), t\right), \\
& w_{2}(t)=\frac{\partial \theta_{2}}{\partial x}\left(s_{2}(t), t\right) .
\end{aligned}
$$


304

PLAIN BENSOUSSAN AND AVNER FRIEDMAN

We apply (4.7) to $u=\theta_{1}$, differentiate with respect to $x$, let $x \rightarrow s_{1}(t)$ or $x \rightarrow s_{2}(t)$ and use a standard jump relation [12], [13, p. 217]. Making use of (4.3)-(4.5), we arrive at the formulas:

$$
\begin{aligned}
& -\frac{1}{2} v_{1}(t)=\int_{-1}^{+1} K\left(s_{1}(t), t ; \xi, 0\right) \dot{g}_{1}(\xi) d \xi \\
& \quad+\int_{0}^{t} K\left(s_{1}(t), t ; s_{2}(\tau), \tau\right)\left[-\lambda_{1 x}\left(s_{2}(\tau), \tau\right) v_{2}(\tau)+\lambda_{1 \tau}\left(s_{2}(\tau), \tau\right)\right] d \tau \\
& \quad+\int_{0}^{t} K_{x}\left(s_{1}(t), t ; s_{1}(\tau), \tau\right) v_{1}(\tau) d \tau \\
& \quad-\int_{0}^{t} K_{x}\left(s_{1}(t), t ; s_{2}(\tau), \tau\right) w_{1}(\tau) d \tau \\
& \quad-\frac{1}{2} w_{1}(t)=\int_{-1}^{+1} K\left(s_{2}(t), t ; \xi, 0\right) \dot{g}_{1}(\xi) d \xi \\
& \quad+\int_{0}^{t} K\left(s_{2}(t), t ; s_{2}(\tau), \tau\right)\left[-\lambda_{1 x}\left(s_{2}(\tau), \tau\right) v_{2}(\tau)+\lambda_{1 \tau}\left(s_{2}(\tau), \tau\right)\right] d \tau \\
& \quad+\int_{0}^{t} K_{x}\left(s_{2}(t), t ; s_{1}(\tau), \tau\right) v_{1}(\tau) d \tau \\
& \quad-\int_{0}^{t} K_{x}\left(s_{2}(t), t ; s_{2}(\tau), \tau\right) w_{1}(\tau) d \tau
\end{aligned}
$$

A similar argument applied to $\theta_{2}$ leads to

$$
\begin{aligned}
\frac{1}{2} v_{2}(t)= & \int_{-1}^{+1} K\left(s_{2}(t), t ; \xi, 0\right) \dot{g}_{2}(\xi) d \xi \\
& -\int_{-1}^{+1} K\left(s_{2}(t), t ; s_{1}(\tau), \tau\right)\left[\lambda_{2 x}\left(s_{1}(\tau), \tau\right) v_{1}(\tau)+\lambda_{2 \tau}\left(s_{1}(\tau), \tau\right)\right] d \tau
\end{aligned}
$$$$
+\int_{0}^{t} K_{x}\left(s_{2}(t), t ; s_{2}(\tau), \tau\right) v_{2}(\tau) d \tau
$$$$
-\int_{0}^{t} K_{x}\left(s_{2}(t), t ; s_{1}(\tau), \tau\right) w_{2}(\tau) d \tau
$$

$$
\frac{1}{2} w_{2}(t)=\int_{-1}^{+1} K\left(s_{1}(t), t ; \xi, 0\right) \dot{g}_{2}(\xi) d \xi
$$

$$
\begin{aligned}
& -\int_{0}^{t} K\left(s_{1}(t), t ; s_{1}(\tau), \tau\right)\left[\lambda_{2 x}\left(s_{1}(\tau), \tau\right) v_{1}(\tau)+\lambda_{2 \tau}\left(s_{1}(\tau), \tau\right)\right] d \tau \\
& +\int_{0}^{t} K_{x}\left(s_{1}(t), t ; s_{2}(\tau), \tau\right) v_{2}(\tau) d \tau \\
& -\int_{0}^{t} K_{x}\left(s_{1}(t), t ; s_{1}(\tau), \tau\right) w_{2}(\tau) d \tau
\end{aligned}
$$


The equations (4.12)-(4.15) form a system of nonlinear integral equations of Volterra type in $v_{1}, v_{2}, w_{1}, w_{2}$, noting that $s_{1}$ and $s_{2}$ are given by

$$
\begin{aligned}
& s_{1}(t)=\int_{0}^{t} v_{1}(\tau) d \tau-1, \\
& s_{2}(t)=-\int_{0}^{t} v_{2}(\tau) d \tau+1 .
\end{aligned}
$$

LEMMA 4.2. There exists a positive number $\sigma$ depending only on $C$ (cf. (4.1) and (4.2)) such that there exists a unique continuous solution $\left(v_{1}(t), v_{2}(t), w_{1}(t)\right.$, $\left.w_{2}(t), s_{1}(t), s_{2}(t)\right)$ of (4.12)-(4.17) for $0 \leqslant t \leqslant \sigma$.

The proof is similar to the proof in the case of the Stefan problem [12].

Suppose now that we have a solution of (4.12)-(4.17). We wish to show that this solution yields a solution of the original free boundary problem (4.3)-(4.6) with

$$
\begin{aligned}
\theta_{1}(x, t)= & \int_{-1}^{+1} K(x, t ; \xi, 0) g_{1}(\xi) d \xi-\int_{0}^{t} v_{2}(\tau) K\left(x, t ; s_{2}(\tau), \tau\right) \lambda_{1}\left(s_{2}(\tau), \tau\right) d \tau \\
& +\int_{0}^{t}\left[-K\left(x, t ; s_{2}(\tau), \tau\right) w_{1}(\tau)+K\left(x, t ; s_{1}(\tau), \tau\right) v_{1}(\tau)\right] d \tau \\
& +\int_{0}^{t} K_{x}\left(x, t ; s_{2}(\tau), \tau\right) \lambda_{1}\left(s_{2}(\tau), \tau\right) d \tau
\end{aligned}
$$

$\theta_{2}(x, t)=\int_{-1}^{+1} K(x, t ; \xi, 0) g_{2}(\xi) d \xi-\int_{0}^{t} v_{1}(\tau) K\left(x, t, s_{1}(\tau), \tau\right) \lambda_{2}\left(s_{1}(\tau), \tau\right) d \tau$

$$
\begin{aligned}
& +\int_{0}^{t}\left[K\left(x, t ; s_{2}(\tau), \tau\right) v_{2}(\tau)-K\left(x, t ; s_{1}(\tau), \tau\right) w_{2}(\tau)\right] d \tau \\
& -\int_{0}^{t} K_{x}\left(x, t ; s_{1}(\tau), \tau\right) \lambda_{2}\left(s_{1}(\tau), \tau\right) d \tau
\end{aligned}
$$

LEMMA 4.3. Let $\left(v_{1}, v_{2}, w_{1}, w_{2}, s_{1}, s_{2}\right)$ be a continuous solution of (4.12)-(4.17) for $0 \leqslant t \leqslant \sigma$ such that $s_{2}(t)-s_{1}(t) \geqslant$ const $>0$. Then $\theta_{1}, \theta_{2}$ defined by (4.18), (4.19) together with $s_{1}, s_{2}$ form a solution of the free boundary value problem (4.3)-(4.6) for $0 \leqslant t \leqslant \sigma$.

The proof is an easy extension of the proof in [12] for the Stefan problem.

Using the maximum principle we can show that the solution of (4.3)-(4.6) asserted in Lemma 4.3 satisfies:

$$
s_{1}(t) \downarrow \text { and } s_{2}(t) \uparrow \text { as } t \text { increases. }
$$

Proof of THeOREM 4.1. We shall prove the following a priori inequalities: For any solution of (4.3)-(4.6) in an interval $0 \leqslant t<t_{0}$

$$
\dot{s}_{1}(t) \geqslant-M \quad\left(0 \leqslant t<t_{0}\right)
$$




$$
\dot{s}_{2}(t) \leqslant N \quad\left(0<t<t_{0}\right)
$$

where $M, N$ are positive constants independent of $t_{0}$.

Using Lemmas 4.2, 4.3 and (4.20) we can then proceed step-by-step to establish the existence of a unique solution of (4.3)-(4.6); the inequalities (4.21), (4.22) guarantee that the $t$-interval in each step is bounded from below by a positive constant; cf. [12].

We shall estimate $\dot{s}_{1}(t)$ at any point $t=\bar{t}$ near $t_{0}, \bar{t}<t_{0}$. Let $R$ be the rectangle given by $s_{1}(\bar{t})<x<1,0<t<\bar{t}$.

Let $w$ be the solution of the heat equation in $R$ satisfying the boundary conditions:

$$
\begin{aligned}
& w=0 \text { for } x=s_{1}(\bar{t}), 0<t<\bar{t}, \\
& w=0 \text { for } t=0 \text { and } s_{1}(\bar{t}) \leqslant x \leqslant-1, \\
& w=g_{1} \text { for } t=0 \text { and }-1 \leqslant x \leqslant 1, \\
& w=k \text { for } x=1,0<t<\bar{t},
\end{aligned}
$$

where $k$ is a constant such that

$$
k>\max _{-1<x<1} g_{1}+\max _{G} \lambda_{1}
$$

where the set $G$ is defined by $s_{1}(\bar{t}) \leqslant x \leqslant s_{2}(\bar{t}), 0 \leqslant t \leqslant \bar{t}$. By the maximum principle $w>\theta_{1}$ for $x=1,0 \leqslant t \leqslant t$. Also $w \geqslant 0$ in $R$; therefore $w \geqslant \theta_{1}$ for $x=s_{1}(t), 0 \leqslant t \leqslant t$. The maximum principle then shows that $w \geqslant \theta_{1}$ in the region $s_{1}(t) \leqslant x \leqslant 1,0 \leqslant t \leqslant \bar{t}$. Noting that $\left(w-\theta_{1}\right)\left(s_{1}(\bar{t}), \bar{t}\right)=0$, we conclude that

$$
\frac{\partial}{\partial x}\left(w-\theta_{1}\right) \geqslant 0 \quad \text { at }\left(s_{1}(\bar{t}), \bar{t}\right)
$$

i.e.,

$$
-\dot{s}_{1}(t)=\frac{\partial \theta_{1}}{\partial x}\left(s_{1}(\bar{t}), \bar{t}\right) \leqslant \frac{\partial w}{\partial x}\left(s_{1}(\bar{t}), \bar{t}\right) .
$$

We also have

$$
\frac{\partial w}{\partial x}\left(s_{1}(\bar{t}), \bar{t}\right) \leqslant M
$$

where $M$ is a constant independent of $\bar{t}$ and of the position of $s_{1}(\bar{t})$. This can be easily checked by using the explicit representation of $w$ in terms of the Green function in the rectangle $R$ (given, for instance, in [13]). The assertion (4.21) follows from (4.23), (4.24). The proof of (4.22) is similar.

4.2. Asymptotic estimates. In this section we give asymptotic estimates on the behavior of function $s_{2}(t)-s_{1}(t)$ as $t \rightarrow \infty$, namely, we shall prove

THEOREM 4.2. Let the assumptions (4.1), (4.2) hold and let 
(4.25) $\quad \varlimsup_{i m} \sup _{x} \lambda_{i}(x, t)<1-\alpha \quad(i=1,2 ; \alpha$ positive constant $)$,

$$
\underset{t \rightarrow \infty}{\lim _{x}}\left\{\inf _{x} \lambda_{1}(x, t)+\inf _{x} \lambda_{2}(x, t)\right\}>\gamma>0 \quad(\gamma \text { constant }) .
$$

Then there exist positive constants $\gamma_{1}, \gamma_{2}$ such that

$$
\gamma_{1} \leqslant \frac{s_{2}(t)-s_{1}(t)}{\sqrt{t}} \leqslant \gamma_{2} \text { for all } t>1 \text {. }
$$

To get the lower bound we actually do not need the condition (4.25); it suffices to assume that the $\lambda_{i}$ are bounded.

Proof. We shall first establish an identity, which is interesting by itself. Multiplying the equation $\theta_{1 x x}=\theta_{1 t}$ by $x-s_{2}(t)$ and integrating over the domain $s_{1}(t)<x<s_{2}(t), 0<t<\sigma$, we get

$$
\int_{0}^{\sigma} \int_{s_{1}(t)}^{s_{2}(t)}\left(x-s_{2}(t)\right) \theta_{1 x x} d x d t=\int_{0}^{\sigma} \int_{s_{1}(t)}^{s_{2}(t)}\left(x-s_{2}(t)\right) \theta_{1 t} d x d t
$$

By integration by parts,

$$
\begin{aligned}
& \int_{0}^{\sigma} d t \int_{s_{1}(t)}^{s_{2}(t)}\left(x-s_{2}(t)\right) \theta_{1 x x} d x \\
& =\int_{0}^{\sigma}\left[\left(s_{1}(t)-s_{2}(t)\right) \dot{s}_{1}(t)-\lambda_{1}\left(s_{2}(t), t\right)\right] d t \\
& \iint\left(x-s_{2}(t)\right) \theta_{1 t}(x, t) d x d t=\int_{s_{1}(\sigma)}^{s_{2}(\sigma)}\left(x-s_{2}(\sigma)\right) \theta_{1}(x, \sigma) d x
\end{aligned}
$$

$$
-\int_{-1}^{1}(x-1) g_{1}(x) d x+\iint \theta_{1}(x, t) \dot{s}_{2}(t) d x d t .
$$

Next, we have

$$
\int_{0}^{\sigma} \int_{s_{1}(t)}^{s_{2}(t)}\left(x-s_{1}(t)\right) \theta_{2 x x} d x d t=\int_{0}^{\sigma} \int_{s_{1}(t)}^{s_{2}(t)}\left(x-s_{1}(t)\right) \theta_{2 t} d x d t
$$

By integration by parts,

$$
\begin{aligned}
& \int_{0}^{\sigma} d t \int_{s_{1}(t)}^{s_{2}(t)}\left(x-s_{1}(t)\right) \theta_{2 x x} d x \\
& =\int_{0}^{\sigma}\left[-\left(s_{2}(t)-s_{1}(t)\right) \dot{s}_{2}(t)+\lambda_{2}\left(s_{1}(t), t\right)\right] d t, \\
& \iint\left(x-s_{1}(t)\right) \theta_{2 t}(x, t) d x d t=\int_{s_{1}(\sigma)}^{s_{2}(\sigma)}\left(x-s_{1}(\sigma)\right) \theta_{2}(x, \sigma) d x \\
& -\int_{-1}^{+1}(x+1) g_{2}(x) d x+\iint \theta_{2}(x, t) \dot{s}_{1}(t) d x d t .
\end{aligned}
$$


Since by (4.28) the right-hand sides of (4.29), (4.30) are equal and, by (4.31), the right-hand sides of (4.32), (4.33) are equal, we get, by adding,

$$
\begin{aligned}
\int_{0}^{\sigma}\left(s_{1}-s_{2}\right)\left(\dot{s}_{1}-\dot{s}_{2}\right) d t & =\int_{0}^{\sigma}\left[\lambda_{1}\left(s_{2}(t), t\right)+\lambda_{2}\left(s_{1}(t), t\right)\right] d t \\
& +\int_{-1}^{+1}\left[(1-x) g_{1}(x)+(1+x) g_{2}(x)\right] d x \\
& -\int_{s_{1}(\sigma)}^{s_{2}(\sigma)}\left[\left(s_{2}(\sigma)-x\right) \theta_{1}(x, \sigma)+\left(x-s_{1}(\sigma)\right) \theta_{2}(x, \sigma)\right] d x \\
& +\iint\left(\theta_{1} \dot{s}_{2}-\theta_{2} \dot{s}_{1}\right) d x d t,
\end{aligned}
$$

or

$\frac{1}{2}\left(s_{2}(\sigma)-s_{1}(\sigma)\right)^{2}=2+\int_{0}^{\sigma}\left(\lambda_{1}+\lambda_{2}\right) d t+\int_{-1}^{+1}\left[(1-x) g_{1}+(1+x) g_{2}\right] d x$

$$
\begin{aligned}
& -\int_{s_{1}(\sigma)}^{s_{2}(\sigma)}\left[\left(s_{2}(\sigma)-x\right) \theta_{1}(x, \sigma)+\left(x-s_{1}(\sigma)\right) \theta_{2}(x, \sigma)\right] d x \\
& +\int_{0}^{\sigma} \int_{s_{1}(t)}^{s_{2}(t)}\left(\theta_{1} \dot{s}_{2}-\theta_{2} \dot{s}_{1}\right) d x d t .
\end{aligned}
$$

Let us now establish the lower bound in (4.27). We note that the double integral in (4.34) is $\geqslant 0$. Since $\theta_{i} \leqslant M(M$ constant), we get from (4.34)

$$
\frac{1}{2}\left(s_{2}(\sigma)-s_{1}(\sigma)\right)^{2}+M\left(s_{2}(\sigma)-s_{1}(\sigma)\right)^{2} \geqslant \gamma \sigma-N
$$

where $\gamma, N$ are positive constants, from which follows the left-hand side of (4.27).

To prove the right-hand side, let $t^{*}$ be such that

$$
\lambda_{i}(x, t) \leqslant 1-\alpha \text { if }-\infty<x<\infty, t \geqslant t^{*} .
$$

We claim that there exists a $t_{0}>t^{*}$ such that

$$
\theta_{i}(x, t) \leqslant 1-\beta, \quad 0<\beta<\alpha \text { if } s_{1}(t)<x<s_{2}(t), t>t_{0} .
$$

Indeed, let $M \geqslant g_{i}(x), M \geqslant 1-\alpha$. Consider the solution $w$ of the heat equation in $-\infty<x<\infty, t>t^{*}$, with initial conditions

$$
w(x, t)= \begin{cases}M & \text { if } s_{1}\left(t^{*}\right)<x<s_{2}\left(t^{*}\right), \\ 1-\alpha & \text { if } x<s_{1}\left(t^{*}\right) \text { or } x>s_{2}\left(t^{*}\right) .\end{cases}
$$

In the domain $s_{1}(t)<x<s_{2}(t), t>t^{*}$ we have, by the maximum principle, $w \geqslant \theta_{i}$. Now, with $s_{i}\left(t^{*}\right)=\alpha_{i}$, 


$$
\begin{aligned}
w(x, t)= & \int_{\alpha_{1}}^{\alpha_{2}} \frac{M}{\left(4 \pi\left(t-t^{*}\right)\right)^{1 / 2}} \exp \left[-\frac{(x-\xi)^{2}}{4\left(t-t^{*}\right)}\right] d \xi \\
& +\int_{\alpha_{2}}^{\infty} \frac{1-\alpha}{\left(4 \pi\left(t-t^{*}\right)\right)^{1 / 2}} \exp \left[-\frac{(x-\xi)^{2}}{4\left(t-t^{*}\right)}\right] d \xi \\
& +\int_{-\infty}^{\alpha_{1}} \frac{1-\alpha}{\left(4 \pi\left(t-t^{*}\right)\right)^{1 / 2}} \exp \left[-\frac{(x-\xi)^{2}}{4\left(t-t^{*}\right)}\right] d \xi
\end{aligned}
$$

Therefore

$$
w(x, t) \leqslant 1-\alpha+M\left(\alpha_{2}-\alpha_{1}\right) /\left(4 \pi\left(t-t^{*}\right)\right)^{1 / 2} .
$$

Taking $t \geqslant t_{0}$ where $t_{0}-t^{*}$ is sufficiently large, we obtain the assertion (4.35).

We can now write an identity similar to (4.34), when $x \in\left(s_{1}(t), s_{2}(t)\right)$, $t_{0} \leqslant t \leqslant \sigma($ instead of $0 \leqslant t \leqslant \sigma)$ :

$$
\begin{aligned}
\frac{1}{2}\left(s_{2}(\sigma)-s_{1}(\sigma)\right)^{2} & =\frac{1}{2}\left(s_{2}\left(t_{0}\right)-s_{1}\left(t_{0}\right)\right)^{2}+\int_{t_{0}}^{\sigma}\left(\lambda_{1}+\lambda_{2}\right) d t \\
& +\int_{s_{1}\left(t_{0}\right)}^{s_{2}\left(t_{0}\right)}\left[\left(s_{2}\left(t_{0}\right)-x\right) \theta_{1}\left(x, t_{0}\right)+\left(x-s_{1}\left(t_{0}\right)\right) \theta_{2}\left(x, t_{0}\right)\right] d x \\
& -\int_{s_{1}(\sigma)}^{s_{2}(\sigma)}\left[\left(s_{2}(\sigma)-x\right) \theta_{1}(x, \sigma)+\left(x-s_{1}(\sigma)\right) \theta_{2}(x, \sigma)\right] d x \\
& +\iint\left(\theta_{1} \dot{s}_{2}-\theta_{2} \dot{s}_{1}\right) d x d t .
\end{aligned}
$$

Using (4.35) we get

$$
\frac{1}{2}\left(s_{2}(\sigma)-s_{1}(\sigma)\right)^{2} \leqslant 2(1-\alpha) \sigma+O(1)+(1-\beta) \int_{t_{0}}^{\sigma}\left(\dot{s}_{2}-\dot{s}_{1}\right)\left(s_{2}-s_{1}\right) d t .
$$

Hence

$$
\beta\left(s_{2}(\sigma)-s_{1}(\sigma)\right)^{2} / 2 \leqslant 2(1-\alpha) \sigma+O(1),
$$

which completes the proof of (4.27).

4.3. The decreasing case. In this section we shall extend Theorem 4.1 to the case where the functions $g_{1}, g_{2}, \lambda_{1}, \lambda_{2}$ are nonpositive. Thus, we assume:

$$
\begin{aligned}
& g_{i}(x) \text { are continuously differentiable and } \leqslant 0 \text { for }-1 \leqslant x \leqslant 1 ; \\
& \left|g_{i x}\right| \leqslant C, g_{1}(-1)=0, g_{2}(1)=0 ; \\
& \quad \lambda_{i}(x, t) \text { are continuously differentiable and } \leqslant 0 ; \\
& \quad \lambda_{i x}|,| \lambda_{i t} \mid \leqslant C, \lambda_{1}(1,0)=g_{1}(1), \lambda_{2}(-1,0)=g_{2}(-1) .
\end{aligned}
$$


We shall also assume:

$$
\begin{aligned}
& \inf _{-1<x<1} g_{1}(x)>-1, \quad \inf _{-1<x<1} g_{2}(x)>-1, \\
& \inf _{-1<x<1, t>0} \lambda_{1}(x, t)>-1, \quad \inf _{-1<x<1, t>0} \lambda_{2}(x, t)>-1 .
\end{aligned}
$$

Let $\lambda_{i}(t), \tilde{\lambda}_{i}(t)$ be functions satisfying $\tilde{\lambda}_{i}(t) \leqslant \lambda_{1}(x, t) \leqslant \lambda_{i}(t) \leqslant 0$ and let

$$
\begin{aligned}
& \Gamma_{1}=-\int_{0}^{\infty} \lambda_{1}(t) d t-\int_{-1}^{1}(1-x) g_{1}(x) d x, \\
& \Gamma_{2}=-\int_{0}^{\infty} \lambda_{2}(t) d t-\int_{-1}^{1}(1+x) g_{2}(x) d x, \\
& \tilde{\Gamma}_{1}=-\int_{0}^{\infty} \tilde{\lambda}_{1}(t) d t-\int_{-1}^{1}(1-x) g_{1}(x) d x, \\
& \tilde{\Gamma}_{2}=-\int_{0}^{\infty} \tilde{\lambda}_{2}(t) d t-\int_{-1}^{1}(1+x) g_{2}(x) d x .
\end{aligned}
$$

Notice that $0 \leqslant \Gamma_{i} \leqslant \tilde{\Gamma}_{i} \leqslant \infty(i=1,2)$.

THEOREM 4.3. Under the assumptions (4.36)-(4.38), there exists a unique solution $\left(\theta_{1}, \theta_{2}, s_{1}, s_{2}\right)$ of (4.3)-(4.6) for all $0 \leqslant t<T^{*}$ for some $T^{*} \in(0, \infty]$; further, $s_{1}(t)$ increases and $s_{2}(t)$ decreases as $t$ increases. If

$$
\Gamma_{1}+\Gamma_{2}>4
$$

then $T^{*}<\infty$, and if

$$
\tilde{\Gamma}_{1}+\tilde{\Gamma}_{2}<2
$$

then $T^{*}=\infty$.

Proof. Let us first show that if there is a solution for $0 \leqslant t<t_{0}$, such that

$$
s_{2}(t)-s_{1}(t) \geqslant \text { const }>0 \text { for } 0 \leqslant t<t_{0} \text {, }
$$

then

$$
\begin{aligned}
& \dot{s}_{1}(t) \leqslant M \quad \text { if } 0 \leqslant t<t_{0}, \\
& -N \leqslant \dot{s}_{2}(t) \quad \text { if } 0 \leqslant t<t_{0},
\end{aligned}
$$

where $M, N$ are positive constants.

To prove (4.42) it is sufficient to verify this inequality at any point $\bar{t}<t_{0}$ with the property that $\dot{s}_{1}(t) \leqslant \dot{s}_{1}(\bar{t})$ if $0 \leqslant t \leqslant \bar{t}$.

We shall need the following lemma.

LemMa 4.4. Let $a, b, \theta, \sigma, t$ be positive numbers such that $\theta=t-\sigma, a<b$. Let $\psi(\tau)=\alpha-a+b(\tau-\sigma)$ where $\alpha$ is a real number and $\tau$ varies in the interval $[\sigma, t]$. Let $W=\{(\xi, \tau) ; \psi(\tau)<\xi<\infty, \sigma<\tau<t\}$. Let $w$ be the bounded solution of 


$$
\begin{aligned}
\frac{\partial w}{\partial \tau} & =\frac{\partial^{2} w}{\partial \xi^{2}} \quad \text { in } W, \\
w(\xi, \sigma) & =0 \quad \text { if } \alpha-a<\xi<\alpha, \\
w(\xi, \sigma) & =k(\xi) \quad \text { if } \alpha<\xi<\infty\left(-k_{0} \leqslant k(\xi) \leqslant 0\right), \\
w(\psi(\tau), \tau) & =0 \quad \text { if } \sigma<\tau<t .
\end{aligned}
$$

Then

$$
0 \leqslant-\frac{\partial w}{\partial \xi}(\psi(t), t)
$$

$$
\leqslant-b \int_{\sigma}^{t} \frac{b \tilde{k}(\tau)}{(4 \pi(t-\tau))^{1 / 2}} \exp \left[-\frac{b^{2}(t-\tau)}{4}\right] d \tau+k_{0} C(\theta)
$$

where

$$
\tilde{k}(\tau)=\int_{\alpha}^{\infty} \frac{k(\xi)}{(4 \pi(\tau-\sigma))^{1 / 2}} \exp \left[-\frac{(\psi(\tau)-\xi)^{2}}{4(\tau-\sigma)}\right] d \xi
$$

and $C(\theta)$ is a constant independent of $a, b, k$.

The proof is given in the Appendix.

Notice that (4.44) implies that

$$
0 \leqslant-\partial w(\psi(t), t) / \partial \xi \leqslant k_{0} b+C(\theta) .
$$

We shall apply Lemma 4.4 with

$$
\begin{gathered}
t=\bar{t}, \sigma=\bar{t}-\delta, b=\dot{s}_{1}(\bar{t}), \alpha=s_{1}(\bar{t}-\delta), s_{1}(\bar{t})=\alpha-a+b \delta, \delta>0, \\
k=\min \left(\inf g_{1}, \inf \lambda_{1}\right)-\varepsilon, \quad-1<k<0 .
\end{gathered}
$$

Notice, by (4.38), that $\varepsilon>0$ can indeed be chosen so that $-1<k<0$. By the maximum principle, $w \leqslant 0$. Hence $w\left(s_{1}(\tau), \tau\right) \leqslant \theta_{1}\left(s_{1}(\tau), \tau\right)$ if $\bar{t}-\delta \leqslant \tau$ $\leqslant \bar{t}$. Next

$$
w(\xi, \bar{t}-\delta)<\theta_{1}(\xi, \bar{t}-\delta) \text { if } s_{1}(\bar{t}-\delta) \leqslant \xi \leqslant s_{2}(\bar{t}-\delta) .
$$

Finally, if $\delta$ is sufficiently small (depending on $\varepsilon$ ) then

$$
w\left(s_{2}(\tau), \tau\right)<\lambda_{1}\left(s_{2}(\tau), \tau\right)=\theta_{1}\left(s_{2}(\tau), \tau\right) \text { for } \bar{t}-\delta<\tau<\bar{t}
$$

By the maximum principle we conclude that $w \leqslant \theta_{1}$ if $s_{1}(\tau)<\xi<s_{2}(\tau)$, $0<\tau<\bar{t}$. Since $w\left(s_{1}(\bar{t}), \bar{t}\right)=\theta_{1}\left(s_{1}(\bar{t}), \bar{t}\right)$, it follows that

$$
\partial\left(\theta_{1}-w\right)\left(s_{1}(\bar{t}), \bar{t}\right) / \partial \xi \geqslant 0 .
$$

Consequently, 


$$
b=\dot{s}_{1}(\bar{t})=-\frac{\partial}{\partial \xi} \theta_{1}\left(s_{1}(\bar{t}), \bar{t}\right) \leqslant-\frac{\partial}{\partial \xi} w\left(s_{1}(\bar{t}), \bar{t}\right) \leqslant-k(b+C(\theta))
$$

by Lemma 4.4, i.e., $(1+k) b \leqslant C(\theta)$. Since $1+k>0$, we get $b<$ const. This completes the proof of (4.42). The proof of (4.43) is similar.

We can now proceed to complete the proof of existence and uniqueness for (4.3)-(4.6) as in the case of Theorem 4.1. As long as (4.41) holds for a constant arbitrarily small, the solution can be continued in a unique way. By letting the constant go to 0 we arrive at a maximal interval $0 \leqslant t<T^{*}$ where the solution of (4.3)-(4.6) exists and is unique. In general, $T^{*} \leqslant \infty$. If $T^{*}<\infty$ then we must have $s_{1}\left(T^{*}-0\right)=s_{2}\left(T^{*}-0\right)$, whereas if $T^{*}=\infty$ then $s_{1}(t)<s_{2}(t)$ for all $0 \leqslant t<\infty$.

We shall now assume that (4.39) holds and prove that $T^{*}<\infty$.

Let $0<\sigma<T^{*}$. Multiplying the heat equation for $\theta_{1}$ by $x-s_{2}(t)$ and integrating with respect to $(x, t)$, over the region $s_{1}(t)<x<s_{2}(t), 0<t<\sigma$, we get

$$
\int d t \int_{s_{1}(t)}^{s_{2}(t)}\left(x-s_{2}(t)\right) \frac{\partial^{2} \theta_{1}}{\partial x^{2}} d x=\int_{-1}^{1} d x \int_{0}^{l(x)}\left(x-s_{2}(t)\right) \frac{\partial \theta_{1}}{\partial t} d t
$$

where

$$
l(x)= \begin{cases}l_{1}(x) & \text { if }-1<x<s_{1}(\sigma), \\ \sigma & \text { if } s_{1}(\sigma)<x<s_{2}(\sigma), \\ l_{2}(x) & \text { if } s_{2}(\sigma)<x<1\end{cases}
$$

and $l_{i}$ is the inverse function to $s_{i}$.

Integrating by parts, we obtain

$$
\begin{aligned}
\int_{0}^{\sigma}\left(s_{1}(t)-s_{2}(t)\right) \dot{s}_{1}(t) d t-\int_{0}^{\sigma} \lambda_{1}\left(s_{2}(t), t\right) d t \\
=\int_{-1}^{1}\left(x-s_{2}(l(x))\right) \theta_{1}(x, l(x)) d x \\
\quad-\int_{-1}^{1}(x-1) g_{1}(x) d x+\int_{-1}^{1} \int_{0}^{l(x)} \dot{s}_{2}(t) \theta_{1}(x, t) d t d x,
\end{aligned}
$$

or,

$$
\begin{aligned}
-\int_{0}^{\sigma} \lambda_{1}\left(s_{2}(t), t\right) d t= & \int_{0}^{\sigma}\left(s_{2}(t)-s_{1}(t)\right) \dot{s}_{1}(t) d t \\
& +\int_{s_{1}(\sigma)}^{s_{2}(\sigma)}\left(x-s_{2}(\sigma)\right) \theta_{1}(x, \sigma) d x \\
& +\int_{-1}^{1}(1-x) g_{1}(x) d x+\int_{0}^{\sigma}\left[\int_{s_{1}(t)}^{s_{2}(t)} \theta_{1}(x, t) d x\right] \dot{s}_{2}(t) d t .
\end{aligned}
$$


By the maximum principle, $-1 \leqslant \theta_{1} \leqslant 0$. Using also the inequalities $\dot{s}_{1} \geqslant 0$, $\dot{s}_{2} \leqslant 0$, we find that the sum of the first and last integrals on the right-hand side of (4.45) is

$$
<\int_{0}^{\sigma}\left(s_{2}(t)-s_{1}(t)\right)\left(\dot{s}_{1}(t)-\dot{s}_{2}(t)\right) d t=2-\frac{1}{2}\left(s_{2}(\sigma)-s_{1}(\sigma)\right)^{2} .
$$

The second integral on the right is

$$
\leqslant \int_{s_{1}(\sigma)}^{s_{2}(\sigma)}\left(s_{2}(\sigma)-x\right) d x=\frac{1}{2}\left(s_{2}(\sigma)-s_{1}(\sigma)\right)^{2} .
$$

Consequently

$$
-\int_{0}^{\sigma} \lambda_{1}\left(s_{2}(t), t\right) d t \leqslant 2+\int_{-1}^{1}(1-x) g_{1}(x) d x .
$$

Similarly,

$$
-\int_{0}^{\sigma} \lambda_{2}\left(s_{1}(t), t\right) d t \leqslant 2+\int_{-1}^{1}(1+x) g_{2}(x) d x .
$$

If $T^{*}=\infty$ then the last two inequalities hold also with $\sigma=\infty$. But then $\Gamma_{1}+\Gamma_{2} \leqslant 4$, which contradicts (4.39).

It remains to prove that if $(4.40)$ holds then $T^{*}=\infty$. Suppose that $T^{*}<\infty$ and take $\sigma \uparrow T^{*}$ in (4.45). Then the second integral on the right converges to zero. Noting that the last integral on the right-hand side of (4.45) is nonnegative, we find that

$$
-\int_{0}^{T} \tilde{\lambda}_{1}(t) d t-\int_{-1}^{1}(1-x) g_{1}(x) d x \geqslant \lim _{\sigma \uparrow T^{*}} \int_{0}^{\sigma}\left(s_{2}(t)-s_{1}(t)\right) \dot{s}_{1}(t) d t
$$

Similarly,

$$
-\int_{0}^{T_{2}} \tilde{\lambda}_{2}(t) d t-\int_{-1}^{1}(1+x) g_{2}(x) d x \geqslant \lim _{\sigma \uparrow T^{*}} \int_{0}^{\sigma}\left(s_{1}(t)-s_{2}(t)\right) \dot{s}_{2}(t) d t .
$$

Since

$$
\int_{0}^{\sigma}\left(s_{2}-s_{1}\right) \dot{s}_{1} d t+\int_{0}^{\sigma}\left(s_{1}-s_{2}\right) \dot{s}_{2} d t=2-\frac{1}{2}\left(s_{2}(\sigma)-s_{1}(\sigma)\right)^{2} \rightarrow 2
$$

as $\sigma \uparrow T^{*}$, we obtain, upon adding (4.46), (4.47), $\tilde{\Gamma}_{1}+\tilde{\Gamma}_{2} \geqslant 2$, which contradicts $(4.40)$.

4.4. The general case. In this section we shall consider the general case where the functions $g_{i}, \lambda_{i}$ need not have the same sign everywhere. More specifically we shall assume:

$$
\begin{aligned}
& g_{i x}(x) \text { are continuously differentiable for }-1 \leqslant x \leqslant 1, \\
& \left|g_{i x}\right| \leqslant C \text {, and } g_{1}(-1)=0, g_{2}(1)=0 ;
\end{aligned}
$$




$$
\begin{aligned}
& \lambda_{i}(x, t) \text { are continuously differentiable and }\left|\lambda_{i x}\right|,\left|\lambda_{i t}\right| \leqslant C ; \\
& \lambda_{1}(1,0)=g_{1}(1), \quad \lambda_{2}(-1,0) g_{2}(-1) \\
& g_{1}\left(\alpha_{i}\right)=0 \quad \text { for } \alpha_{0}=-1<\alpha_{1}<\alpha_{2}<\cdots<\alpha_{k}<1, \\
& g_{1}(x) \neq 0 \quad \text { if }-1<x<1 \text { and } x \neq \alpha_{i} \text { for all } i, \\
& g_{1}(x) \text { changes sign as } x \text { crosses } \alpha_{i}(1 \leqslant i \leqslant k), \\
& g_{2}\left(\beta_{i}\right)=0 \text { for } \beta_{0}=1>\beta_{1}>\beta_{2}>\cdots>\beta_{l}>-1, \\
& g_{2}(x) \neq 0 \quad \text { if }-1<x<1 \text { and } x \neq \beta_{i} \text { for all } i, \\
& g_{2}(x) \text { changes sign as } x \text { crosses } \beta_{i}(1 \leqslant i \leqslant l) .
\end{aligned}
$$

THEOREM 4.4. Under the assumptions (4.48)-(4.50) and (4.38), there exists a unique solution $\left(\theta_{1}, \theta_{2}, s_{1}, s_{2}\right)$ of (4.3)-(4.6) for all $0 \leqslant t<T$ for some $T \in(0$, $\infty)$; further $s_{1}(t)$ and $s_{2}(t)$ are both piecewise monotone functions, and $s_{2}(T-0)$ $=s_{1}(T-0)$ in case $T<\infty$.

From the proof it will follow that the direction of monotonicity of $s_{1}(t)$ $\left(s_{2}(t)\right)$ changes at most $k(l)$ times.

Proof. One can prove the local existence and uniqueness of a solution in precisely the same way as in Theorem 4.1. Thus, all that remains to be shown is the piecewise monotonicity of $s_{i}(t)$ and the a priori bound on $\dot{s}_{i}(t)$.

Denote by $\gamma_{i}$ the curve defined by $\theta_{1}=0$, initiating at $\left(\alpha_{i}, 0\right)$; these curves are constructed in Friedman [16] and Van Moerbeke [24].

Suppose $g_{1}(x)>0$ if $-1<x<\alpha_{1}$. We claim that as long as $\gamma_{1}$ does not intersect $x=s_{1}(t)$ the function $s_{1}(t)$ is monotone decreasing. Indeed, by the maximum principle applied to $\theta_{1}$ in the region bounded by $x=s_{1}(t), \gamma_{1}$ and $t=0$ we find that $\theta_{1}$ takes its minimum on the boundary $x=s_{1}(t)$. Hence

$$
\dot{s}_{1}(t)=-\theta_{1 x}\left(s_{1}(t), t\right) \leqslant 0 .
$$

One can derive a priori bound on $\dot{s}_{1}(t)$ (as long as $x=s_{1}(t)$ does not intersect $\gamma_{1}$ ) by the method of $\$ 4.1$.

Consider next the case where $g_{1}(x)<0$ if $-1<x<\alpha_{1}$. Using an argument similar to one given above we deduce that $\dot{s}_{1}(t) \geqslant 0$ as long as $x=s_{1}(t)$ does not intersect $\gamma_{1}$. To find a priori bound on $\dot{s}_{1}(t)$ we use Lemma 4.4 (as in the proof of Theorem 4.3). However, here we may simply take $\sigma=0$, i.e., $\delta=\bar{t}$.

Denote by $t_{1}$ the first time $\gamma_{1}$ intersects $x=s_{1}(t)$. For $t>t_{1}$ we have to take into account the curve $\gamma_{2}$. As long as $\gamma_{2}$ does not intersect $x=s_{1}(t)$, the function $s_{1}(t)$ is monotone (with the direction of monotonicity reversed to the direction of monotonicity in the interval $0<t<t_{1}$ ). Furthermore, one can estimate $\dot{s}_{1}(t)$ as before. If $\gamma_{2}$ intersects $x=s_{1}(t)$ at time $t_{2}$, then for $t>t_{2}$ we have to consider the curve $\gamma_{3}$; etc.

The monotone behavior of $s_{2}(t)$ and the a priori bounds on $\dot{s}_{2}(t)$ can be obtained in a similar manner. 
Notice that as long as $s_{2}(t)-s_{1}(t)$ remains positive, we can continue with the construction of the solution step-by-step in $t$. After time $t=t_{k_{0}}$ when the last $\gamma_{k_{0}}$ intersects $x=s_{1}(t)\left(1 \leqslant k_{0} \leqslant k\right)$, the derivation of the a priori bound on $s_{1}(t)$ is slightly different than before, in the monotone decreasing case. Indeed, when we apply Lemma 4.4 we now have to take $t=\bar{t}, \sigma=\bar{t}-\delta$ with $\delta$ sufficiently small (cf. the proof of Theorem 4.3). The same discussion applies to the curve $x=s_{2}(t)$.

\section{Chapter 5. Zero-Sum Stochastic Game with Stopping Times}

\subsection{Formal derivation of the Stefan problem. For zero-sum game,}

$$
f_{2}=-f_{1}=-f, \quad \phi_{2}=-\psi_{1}=-\phi, \quad \psi_{2}=-\phi_{1}=-\phi .
$$

This case has been already studied by Friedman [14], as far as the existence and uniqueness are concerned. We want here to relate this problem to the methods of Chapter 4 and study the corresponding Stefan problem.

Let $Q=\{(x, t) ;-\infty<x<\infty, 0<t<T\}$ and consider a function $u(x$, $t)$ satisfying

$$
\begin{aligned}
& u \text { is continuous in } \bar{Q},\left(u_{t}+u_{x x}\right) \in L_{\mathrm{loc}}^{2}(Q), \\
& \psi \leqslant u \leqslant \phi \text { in } Q, \\
& \text { if } u>\psi \text { then }-u_{t}-u_{x x} \leqslant f, \\
& \text { if } u<\phi \text { then }-u_{t}-u_{x x} \geqslant f, \\
& u(x, T)=h(x) \text { if }-\infty<x<\infty .
\end{aligned}
$$

We assume throughout this section that $\phi, \psi$ are in $C^{2}(\bar{Q}), f$ is in $C(\bar{Q}), h$, $h^{\prime}$ are continuous for all $x$, and $h^{\prime \prime}$ is continuous in $[-1,1]$. It is easy to check that $u_{1}=u$ and $u_{2}=-u$ satisfy all the sufficient conditions of Theorem 1.1; consequently, a solution of (5.1)-(5.5) provides a Nash equilibrium point.

The existence and uniqueness of the solution of (5.1)-(5.5) follows from the general theory of parabolic variational inequalities of Lions-Stampacchia [21] (see also [14], [15]). The connection between parabolic variational inequalities and Stefan problems has been developed by Duvaut [11], Friedman [16] and Friedman and Kinderlehrer [19]; all these authors worked with one-sided inequality.

We shall first derive formally the Stefan problem corresponding to (5.1)-(5.5). For convenience, we shall change the time from $t$ into $T-t$. If $u$ satisfies:

$$
\begin{aligned}
& u \text { is continuous in } \bar{Q},\left(u_{t}-u_{x x}\right) \in L_{\mathrm{loc}}^{2}(Q), \\
& \psi \leqslant u \leqslant \phi \\
& \text { if } u>\psi \text { then } u_{t}-u_{x x} \leqslant f, \\
& \text { if } u<\phi \text { then } u_{t}-u_{x x} \geqslant f, \\
& u(x, 0)=h(x),
\end{aligned}
$$


then $u(x, T-t)$ satisfies (5.1)-(5.5) with $f(x, t), \phi(x, t), \psi(x, t)$ replaced by $f(x, T-t), \phi(x, T-t), \psi(x, T-t)$.

Let $s_{1}(t)$ and $s_{2}(t)$ be the two boundaries separating respectively $u<\phi$ from $u=\phi$ and $u>\psi$ from $u=\psi$ respectively. Let us assume that these functions satisfy

$$
\begin{gathered}
s_{1}(t)<s_{2}(t), s_{1}(0)=-1, s_{2}(0) 1, \\
\dot{s}_{1} \leqslant 0, \dot{s}_{2} \geqslant 0 .
\end{gathered}
$$

Let $l_{1}(x), l_{2}(x)$ be the inverse functions of $s_{1}(t), s_{2}(t)$ respectively. We define $\theta(x, t)$ by the following relations:

$$
\begin{aligned}
\theta & =0 \text { if } x \leqslant-1, t \leqslant l_{1}(x), \\
\phi-u & =\int_{l_{1}(x)}^{t} \theta(x, \tau) d \tau \quad \text { if } x \leqslant 1, t \geqslant l_{1}(x), \\
\phi-u & =\int_{0}^{t} \theta(x, \tau) d \tau-h(x) \quad \text { if }-1 \leqslant x \leqslant 1, t>0, \\
\phi-u & =\int_{l_{2}(x)}^{t} \theta(x, \tau) d \tau+\phi\left(x, l_{2}(x)\right)-\psi\left(x, l_{2}(x)\right) \\
\theta & =\phi_{t}-\psi_{t} \text { if } x \geqslant 1, t \geqslant l_{2}(x),
\end{aligned}
$$

One easily checks that

$$
\phi_{t}-u_{t}=\theta
$$

We shall assume:

$$
\begin{gathered}
h=0 \text { for } x \leqslant-1, \quad h \leqslant 0 \quad \text { if } x \geqslant-1 ; \\
h(x) \geqslant \psi(x, 0) \text { for } x \in[-1,+1], \\
h(x)=\psi(x, 0) \text { for } x \geqslant 1, \\
\phi(x, 0) \equiv 0 \quad \text { for } x \geqslant 1 ; \\
\quad f-\phi_{t}+\phi_{x x}=1 .
\end{gathered}
$$

From the regularity conditions (5.6) it follows that a.e. in $t, u_{x}$ is continuous in $x$. Therefore on the free boundaries we have

$$
\begin{aligned}
& u_{x}\left(s_{1}(t), t\right)=\phi_{x}\left(s_{1}(t), t\right), \\
& u_{x}\left(s_{2}(t), t\right)=\psi_{x}\left(s_{2}(t), t\right) .
\end{aligned}
$$

Differentiating (5.14) in $x$ and taking $t=l_{1}(x)$, we get, taking (5.22) into account,

$$
\theta\left(s_{1}(t), t\right)=0
$$


Differentiating (5.16) with respect to $x$, we get

$$
\begin{aligned}
\phi_{x}(x, t)-u_{x}(x, t)= & -i_{2}(x) \theta\left(x, l_{2}(x)\right)+\left(\phi_{x}-\psi_{x}\right)\left(x, l_{2}(x)\right) \\
& +i_{2}(x)\left(\phi_{t}-\psi_{t}\right)\left(x, l_{2}(x)\right)+\int_{l_{2}(x)}^{t} \theta_{x}(x, \tau) d \tau .
\end{aligned}
$$

Taking $t=l_{2}(x)$ and using (5.23), we get

$$
\theta\left(s_{2}(t), t\right)=\left(\phi_{t}-\psi_{t}\right)\left(s_{2}(t), t\right) .
$$

In the region where (5.14) holds, we get by differentiating twice in $x$,

$$
\left(\phi_{x x}-u_{x x}\right)(x, t)=-i_{1}(x) \theta_{x}\left(x, l_{1}(x)\right)+\int_{l_{1}(x)}^{t} \theta_{x x}(x, \tau) d \tau .
$$

Therefore, using the fact that $\theta\left(x, l_{1}(x)\right)=0$ (i.e., (5.24)) we get, taking (5.21) into account,

$$
1=-i_{1}(x) \theta_{x}\left(x, l_{1}(x)\right)-\int_{l_{1}(x)}^{t}\left(\theta_{\tau}-\theta_{x x}\right)(x, \tau) d \tau
$$

from which we deduce

$$
\begin{gathered}
\theta_{x}\left(s_{1}(t), t\right)=-\dot{s}_{1}(t), \\
\theta_{1}-\theta_{x x}=0 \quad \text { if } x<-1, t>l_{1}(x) .
\end{gathered}
$$

From (5.15) we deduce by similar calculations

hence

$$
1+\int_{0}^{t}\left(\theta_{\tau}-\theta_{x x}\right)(x, \tau) d \tau+\theta(x, 0)=-h^{\prime \prime}(x)
$$

$$
\begin{array}{ll}
\theta(x, 0)=-\left(1+h^{\prime \prime}(x)\right) & \text { if } x \in(-1,+1) \\
\theta_{t}-\theta_{x x}=0 & \text { if } x \in(-1,+1), t>0 .
\end{array}
$$

Next, using (5.26) in (5.25), and differentiating (5.25) with respect to $x$, we get

$$
\begin{aligned}
\left(\phi_{x x}-u_{x x}\right)(x, t)= & \left(\phi_{x x}-\psi_{x x}\right)\left(x, l_{2}(x)\right)+i_{2}(x)\left(\phi_{x t}-\psi_{x t}\right)\left(x, l_{2}(x)\right) \\
& -i_{2}(x) \theta_{x}\left(x, l_{2}(x)\right)+\int_{l_{2}(x)}^{t} \theta_{x x}(x, \tau) d \tau .
\end{aligned}
$$

After some rearrangement,

$$
\begin{aligned}
1+\left(\phi_{t}-\psi_{t}-\left(\phi_{x x}-\right.\right. & \left.\left.\psi_{x x}\right)\right)\left(x, l_{2}(x)\right)+\int_{l_{2}(x)}^{t}\left(\theta_{\tau}-\theta_{x x}\right)(x, \tau) d \tau \\
& =i_{2}(x)\left(\left(\phi_{x t}-\psi_{x t}\right)\left(x, l_{2}(x)\right)-\theta_{x}\left(x, l_{2}(x)\right)\right)
\end{aligned}
$$

Setting

$$
\lambda=\phi_{t}-\psi_{t}, \quad-\mu=1+\phi_{t}-\psi_{t}-\phi_{x x}+\psi_{x x},
$$

we get from (5.31) 


$$
\begin{gathered}
\theta_{x}\left(s_{2}(t), t\right)-\lambda_{x}\left(s_{2}(t), t\right)=\dot{s}_{2}(t) \mu\left(s_{2}(t), t\right), \\
\theta_{t}-\theta_{x x}=0 \quad \text { if } x>1, t>l_{2}(x) .
\end{gathered}
$$

5.2. Verification theorem. We can now state our Stefan problem: find functions $\theta(x, t), s_{1}(t), s_{2}(t)$ such that

$$
\begin{aligned}
& s_{1}(t)<s_{2}(t), \quad s_{1}(0)=-1, \quad s_{2}(0)=+1, \\
& \partial \theta / \partial t-\partial^{2} \theta / \partial x^{2}=0 \quad \text { if } x \in\left(s_{1}(t), s_{2}(t)\right), t>0, \\
& \theta\left(s_{1}(t), t\right)=0, \quad t \geqslant 0, \\
& \theta\left(s_{2}(t), t\right)=\lambda\left(s_{2}(t), t\right), \quad t \geqslant 0, \\
& \theta_{x}\left(s_{1}(t), t\right)=-\dot{s}_{1}(t), \quad t \geqslant 0, \\
& \theta_{x}\left(s_{2}(t), t\right)=\lambda_{x}\left(s_{2}(t), t\right)+\mu\left(s_{2}(t), t\right) \dot{s}_{2}(t), \quad t \geqslant 0, \\
& \theta(x, 0)=g(x), \quad x \in[-1,+1], \\
& s_{1}, s_{2} \text { are continuously differentiable for } t \geqslant 0, \\
& \theta, \theta_{x}, \theta_{t} \text { are continuous in }(x, t), \\
& \theta_{x x} \text { is continuous for } x \in\left(s_{1}(t), s_{2}(t)\right), t>0 .
\end{aligned}
$$

We shall make the following assumptions:

$$
g(x)=-\left(1+h^{\prime \prime}(x)\right) \geqslant 0, \quad g(-1)=0,
$$

$g$ is continously differentiable in $[-1,+1]$;

(5.44) $\lambda$ is continuously differentiable, $\quad\left|\lambda_{x}\right| \leqslant C, \quad\left|\lambda_{t}\right| \leqslant C, \quad \lambda \geqslant 0$;

(5.45) $\lambda(x, 0) \geqslant g(x) \quad$ if $-1 \leqslant x \leqslant 1, \quad \lambda(1,0)=g(1)$;

(5.46) $0<\alpha \leqslant \mu \leqslant C_{0}, \mu_{t} \leqslant 0 \quad\left(\alpha, C_{0}\right.$ positive constants).

Suppose now that there exists a solution of (5.35)-(5.42). Since $g \geqslant 0, \lambda \geqslant 0$, it follows from the maximum principle that $\theta \geqslant 0$. Since $\theta=0$ on $x=s_{1}(t)$, we see that $\theta_{x}\left(s_{1}(t), t\right) \geqslant 0$; hence from (5.39) we get

$$
\dot{s}_{1}(t) \leqslant 0 \text {. }
$$

Consider next the function $w=\theta-\lambda$. Clearly, $w=0$ on $\left(s_{2}(t), t\right), w=-\lambda$ $\leqslant 0$ on $\left(s_{1}(t), t\right)$ and $w=g-\lambda(x, 0) \leqslant 0$ for $t=0$. Since

$$
w_{t}-w_{x x}=\theta_{t}-\theta_{x x}-\lambda_{t}+\lambda_{x x}=\mu_{t} \leqslant 0,
$$

it follows from the maximum principle that $w \leqslant 0$. Since $w=0$ on $\left(s_{2}(t)\right.$, $t$ ), we have

$$
\left(\theta_{x}-\lambda_{x}\right)\left(s_{2}(t), t\right) \geqslant 0 .
$$

Since $\mu>0$, we get from (5.40) that

$$
\dot{s}_{2}(t) \geqslant 0 \text {. }
$$

We next define $u(x, t)$ from $\theta(x, t)$ by using formulas (5.14)-(5.16) and 


$$
\begin{aligned}
& u=\phi \text { for } x \leqslant-1, t \leqslant l_{1}(x), \\
& u=\psi \text { for } x \geqslant 1, t \leqslant l_{2}(x),
\end{aligned}
$$

and check that it is indeed a solution of (5.6)-(5.10). Clearly $u$ is continuous in $\bar{Q}$. We next have $\phi_{t}-u_{t}=\theta$ which proves that $u_{t}$ is continuous. Next

$$
\begin{aligned}
u_{x} & =\phi_{x} \text { for } x \leqslant-1, t \leqslant l_{1}(x), \\
\phi_{x}-u_{x} & =\int_{l_{1}(x)}^{t} \theta_{x}(x, \tau) d \tau \text { for } x \leqslant-1, t \geqslant l_{1}(x), \\
\phi_{x}-u_{x} & =\int_{0}^{t} \theta_{x}(x, \tau) d \tau-h^{\prime}(x) \quad \text { for }-1 \leqslant x \leqslant 1, t \geqslant 0, \\
\phi_{x}-u_{x} & =\int_{l_{2}(x)}^{t} \theta_{x}(x, \tau) d \tau+\phi_{x}\left(x, l_{2}(x)\right)-\psi_{x}\left(x, l_{2}(x)\right) \\
u_{x} & =\psi_{x} \text { for } x \geqslant 1, t \leqslant l_{2}(x) ; \quad \text { for } x \geqslant 1, t \geqslant l_{2}(x),
\end{aligned}
$$

it follows that $u_{x}$ is continuous. Next,

$$
\begin{aligned}
u_{x x} & =\phi_{x x} \text { for } x<-1, t<l_{1}(x) \\
\phi_{x x}-u_{x x} & =1+\int_{l_{1}(x)}^{t} \theta_{x x}(x, \tau) d \tau \text { for } x<-1, t>l_{1}(x) \\
\phi_{x x}-u_{x x} & =\int_{0}^{t} \theta_{x x}(x, \tau) d \tau-h^{\prime \prime}(x) \text { for }-1<x<1, t>0, \\
\phi_{x x}-u_{x x} & =\int_{l_{2}(x)}^{t} \theta_{x x}(x, \tau) d \tau+1+\left(\phi_{t}-\psi_{t}\right)\left(x, l_{2}(x)\right)
\end{aligned}
$$

$$
\text { for } x>1, t>l_{2}(x)
$$

$$
u_{x x}=\psi_{x x} \text { for } x>1, t>l_{2}(x)
$$

from which it easily follows that $u_{x x} \in L_{\text {loc }}^{2}$. Since $\theta \geqslant 0$ and $h \leqslant 0, \phi \geqslant \psi$, formulas (5.14)-(5.16) and (5.49), (5.50) imply that $u \leqslant \phi$.

Now from above we know that $w=\theta-\lambda \leqslant 0$; hence

$$
\left(u-\psi_{t}\right)=\phi_{t}-\theta-\psi_{t}=\lambda-\theta \geqslant 0 .
$$

But $(u-\psi)\left(s_{1}(t), t\right) \geqslant 0, \quad(u-\psi)\left(s_{2}(t), t\right)=0, \quad(u-\psi)(x, 0)=h-$ $\psi(x, 0) \geqslant 0$ for $x \in[-1,1]$. Therefore $u-\psi \geqslant 0$ for $s_{1}(t) \leqslant x \leqslant s_{2}(t)$, $t \geqslant 0$. From formulas (5.52), (5.53), (5.54) we get

$$
u_{t}-u_{x x}=f \text { if } s_{1}(t)<x<s_{2}(t), t>0,
$$

and

$$
\begin{aligned}
& u_{t}-u_{x x}-f=\phi_{t}-\phi_{x x}-f=-1 \leqslant 0 \text { if } x<-1, t<l_{1}(x) \\
& u_{t}-u_{x x}-f=\psi_{t}-\psi_{x x}-f=\mu \geqslant 0 \text { if } x>1, t<l_{2}(x) .
\end{aligned}
$$


Hence (5.8), (5.9) are proved. Finally (5.10) follows from the definition of $u$ and from the assumptions on $h$. We have thus proved

THEOREM 5.1. Under the assumptions (5.19)-(5.21) and (5.43)-(5.46), a solution $\theta, s_{1}, s_{2}$ of (5.35)-(5.42) yields by formulas (5.14)-(5.16) and (5.49), (5.50) a solution of (5.6)-(5.10).

\subsection{Solution of the Stefan problem. Setting}

$$
v_{1}(t)=\dot{s}_{1}(t), \quad v_{2}(t)=-\dot{s}_{2}(t)
$$

we can derive integral equations for $v_{1}, v_{2}$ in a way similar to what we did in Chapter 4. Using the general formula (4.7) with $u=\theta$ we get, taking into account (5.37)-(5.40),

$$
\begin{aligned}
\theta(x, t)= & \int_{-1}^{+1} K(x, t ; \xi, 0) g(\xi) d \xi-\int_{0}^{t} v_{2}(\tau) K\left(x, t ; s_{2}(\tau), \tau\right) \lambda\left(s_{2}(\tau), \tau\right) d \tau \\
(5.58) \quad & +\int_{0}^{t} K\left(x, t ; s_{2}(\tau), \tau\right)\left[\lambda_{x}\left(s_{2}(\tau), \tau\right)-v_{2}(\tau) \mu\left(s_{2}(\tau), \tau\right)\right] d \tau \\
& +\int_{0}^{t} K\left(x, t ; s_{1}(\tau), \tau\right) v_{1}(\tau) d \tau-\int_{0}^{t} \lambda\left(s_{2}(\tau), \tau\right) \frac{\partial K}{\partial \xi}\left(x, t ; s_{2}(\tau), \tau\right) d \tau
\end{aligned}
$$

Differentiating in $x$ and letting $x \rightarrow s_{1}(t), s_{2}(t)$ respectively we get (cf. the derivation of (4.12))

$-\frac{1}{2} v_{1}(t)=\int_{-1}^{+1} K\left(s_{1}(t), t ; \xi, 0\right) \dot{g}(\xi) d \xi$

$$
\begin{aligned}
& +\int_{0}^{t} K_{x}\left(s_{1}(t), t ; s_{2}(\tau), \tau\right)\left[\lambda_{x}\left(s_{2}(\tau), \tau\right)-\mu\left(s_{2}(\tau), \tau\right) v_{2}(\tau)\right] d \tau \\
& +\int_{0}^{t} K_{x}\left(s_{1}(t), t ; s_{1}(\tau), \tau\right) v_{1}(\tau) d \tau \\
& +\int_{0}^{t} K\left(s_{1}(t), t ; s_{2}(\tau), \tau\right)\left[-\lambda_{x}\left(s_{2}(\tau), \tau\right) v_{2}(\tau)+\lambda_{t}\left(s_{2}(\tau), \tau\right)\right] d \tau
\end{aligned}
$$

$\frac{1}{2}\left[\lambda_{x}\left(s_{2}(t), t\right)-v_{2}(t) \mu\left(s_{2}(t), t\right)\right]$

$$
=\int_{-1}^{+1} K\left(s_{2}(t), t ; \xi, 0\right) \dot{g}(\xi) d \xi
$$

$$
\begin{aligned}
& +\int_{0}^{t} K_{x}\left(s_{2}(t), t ; s_{2}(\tau), \tau\right)\left[\lambda_{x}\left(s_{2}(\tau), \tau\right)-\mu\left(s_{2}(\tau), \tau\right) v_{2}(\tau)\right] d \tau \\
& +\int_{0}^{t} K_{x}\left(s_{2}(t), t ; s_{1}(\tau), \tau\right) v_{1}(\tau) d \tau \\
& +\int_{0}^{t} K\left(s_{2}(t), t ; s_{2}(\tau), \tau\right)\left[-\lambda_{x}\left(s_{2}(\tau), \tau\right) v_{2}(\tau)+\lambda_{t}\left(s_{2}(\tau), \tau\right)\right] d \tau
\end{aligned}
$$


Since $\mu \geqslant \alpha>0$, equations (5.59), (5.60) are two nonlinear integral equations of Volterra type, when one adds

$$
\begin{aligned}
& s_{1}(t)=\int_{0}^{t} v_{1}(\tau) d \tau-1, \\
& s_{2}(t)=1-\int_{0}^{t} v_{2}(\tau) d \tau .
\end{aligned}
$$

Existence uniqueness and monotonicity properties of the free boundaries can be established by the methods of $\$ 4.1$. Hence

THEOREM 5.2. Under the assumptions of Theorem 5.1, there exists a unique solution of (5.59)-(5.62). Defining $\theta$ by (5.58), the triple $\left(\theta, s_{1}, s_{2}\right)$ is a solution of the Stefan problem (5.35)-(5.42).

RemarK 1. Theorem 4.2 can be extended to the present case. Thus, in particular, if

$$
\mu \geqslant 1, \quad \varlimsup_{t \rightarrow \infty} \sup _{x} \lambda(x, t)<1,
$$

then there is a positive constant $\gamma$ such that

$$
\frac{s_{2}(t)-s_{1}(t)}{\sqrt{t}} \leqslant \gamma \text { if } t \geqslant 0 .
$$

The proof is obtained from an identity similar to (4.34).

REMARK 2. Theorems $4.3,4.4$ extend to the case where the function $g(x)$ takes also negative values. Here we need to apply the methods of $\S \$ 4.3,4.4$. In case $\lambda \equiv$ const, Lemma 4.4 can be applied and the extension of Theorems 4.3, 4.4 does not present any difficulties.

\section{Appendix: Proof of Lemma 4.4}

By Green's formula

$$
w(x, s)=\int_{\alpha}^{\infty} K(x, s ; \xi, \sigma) k(\xi) d \xi+\int_{\sigma}^{s} K(x, s ; \psi(\tau), \tau) v(\tau) d \tau
$$

where $v(\tau)=-\partial w(\psi(\tau), \tau) / \partial \xi$. The condition $w(\psi(s), s)=0$ can be expressed in the form

$$
\int_{\sigma}^{s} K(\psi(s), s ; \psi(\tau), \tau) v(\tau) d \tau
$$

$$
=-\int_{\alpha}^{\infty} \frac{k(\xi)}{2 \pi^{1 / 2}(s-\sigma)^{1 / 2}} \exp \left\{-\frac{(\psi(s)-\xi)^{2}}{4(s-\sigma)}\right\} d \xi=\frac{f(s)}{2 \pi^{1 / 2}}
$$

i.e., 
(A.3)

$$
\int_{\sigma}^{s} \frac{1}{(s-\tau)^{1 / 2}} \exp \left\{-\frac{b^{2}}{4}(s-\tau)\right\} v(\tau) d \tau=f(s) .
$$

Note that $f(\sigma)=0$.

By Abel's method (see Van Moerbeke [24]) we can solve $v(\tau)$ in terms of $f(s)$ as follows: We multiply both sides of (A.3) by $(t-s)^{1 / 2} \exp \left(b^{2} s / 4\right)$ and integrate with respect to $s, \sigma<s<t$, and then differentiate both sides with respect to $t$. We find that

$$
v(s)=\frac{1}{\pi} \int_{\sigma}^{s} \frac{1}{(s-\tau)^{1 / 2}} \exp \left(-\frac{b^{2} s}{4}\right) \frac{d}{d \tau}\left\{\exp \left(\frac{b^{2} \tau}{4}\right) f(\tau)\right\} d \tau,
$$

i.e.,

$$
\begin{aligned}
v(s)= & \frac{b^{2}}{4 \pi} \int_{\sigma}^{s} \frac{f(\tau)}{(s-\tau)^{1 / 2}} \exp \left\{-\frac{b^{2}(s-\tau)}{4}\right\} d \tau \\
& +\frac{1}{\pi} \int_{\sigma}^{s} \frac{f^{\prime}(\tau)}{(s-\tau)^{1 / 2}} \exp \left\{-\frac{b^{2}(s-\tau)}{4}\right\} d \tau \\
= & I_{1}(s)+I_{2}(s) .
\end{aligned}
$$

By direct computation we find that $I_{1}(t)$ is equal to the first term on the right-hand side of the second inequality of (4.44). Hence it remains to prove that

$$
I_{2}(t) \leqslant k_{0} C(\theta)
$$

To prove (A.6) we compute

$$
\begin{array}{r}
f^{\prime}(\tau)=-\int_{\alpha}^{\infty} k(\xi)\left\{-\frac{1}{2}(\tau-\sigma)^{-3 / 2}+\frac{1}{4}(\tau-\sigma)^{-5 / 2}(\xi-\psi(\tau))^{2}\right. \\
\left.+\frac{b}{2}(\tau-\sigma)^{-3 / 2}(\xi-\psi(\tau))\right\} \\
\cdot \exp \left[-\frac{(\xi-\psi(\tau))^{2}}{4(\tau-\sigma)}\right] d \xi .
\end{array}
$$

In the last braces, the first term is negative and the other two terms are positive. Hence

$$
\begin{array}{r}
f^{\prime}(\tau) \leqslant k_{0} \int_{\alpha}^{\infty}\left\{\frac{1}{4}(\tau-\sigma)^{-5 / 2}(\xi-\psi(\tau))^{2}+\frac{b}{2}(\tau-\sigma)^{-3 / 2}(\xi-\psi(\tau))\right\} \\
\cdot \exp \left[-\frac{(\xi-\psi(\tau))^{2}}{4(\tau-\sigma)}\right] d \xi .
\end{array}
$$


By integration by parts,

$$
\begin{aligned}
f^{\prime}(\tau) \leqslant \frac{1}{2} k_{0}\left\{\frac{1}{2}(\tau-\sigma)^{-1} \int_{\alpha}^{\infty} \exp \left[-\frac{(\xi-\psi(\tau))^{2}}{4(\tau-\sigma)}\right] d \xi\right. \\
+2\left[b(\tau-\sigma)^{1 / 2}+\frac{1}{2}(\tau-\sigma)^{-1}(\alpha-\psi(\tau))\right] \\
\left.\cdot \exp \left[-\frac{(\alpha-\psi(\tau))^{2}}{4(\tau-\sigma)}\right]\right\} .
\end{aligned}
$$

Setting $\tau-\sigma=x$ we get

$$
\begin{aligned}
& f^{\prime}(\sigma+x) \\
& \quad<\frac{1}{2} k_{0}\left\{2 \pi^{1 / 2} x^{1 / 2}+2\left[b x^{-1 / 2}+\frac{1}{2} x^{-1}(a-b x)\right] \exp \left[-\frac{(a-b x)^{2}}{4 x}\right]\right\} .
\end{aligned}
$$

Substituting this into $I_{2}(t)$ we obtain

$$
\begin{aligned}
I_{2}(t) \leqslant & \frac{k_{0}}{\pi^{1 / 2}} \int_{0}^{\theta} \frac{x^{1 / 2}}{(\theta-x)^{1 / 2}} \exp \left[-\frac{b^{2}(\theta-x)}{4}\right] d x \\
& +\frac{k_{0}}{2 \pi} \int_{0}^{\theta} \frac{a}{x(\theta-x)^{1 / 2}} \exp \left[-\frac{(a-b x)^{2}}{4 x}\right] \exp \left[-\frac{b^{2}(\theta-x)}{4}\right] d x \\
& +\frac{k_{0} b}{\pi} \int_{0}^{\theta} \frac{1}{x^{1 / 2}(\theta-x)^{1 / 2}} \exp \left[-\frac{(a-b x)^{2}}{4 x}\right] \exp \left[-\frac{b^{2}(\theta-x)}{4}\right] d x \\
& \equiv J_{1}+J_{2}+J_{3} .
\end{aligned}
$$

We have

$$
J_{1} \leqslant \frac{2 k_{0}}{\pi^{1 / 2}}
$$

Next,

$$
\begin{aligned}
J_{2} & =\frac{k_{0} a}{2 \pi} \int_{0}^{\theta} \frac{1}{x(\theta-x)^{1 / 2}} \exp \left[-\frac{1}{4}\left(b^{2} \theta-2 a b+\frac{a^{2}}{x}\right)\right] d x \\
& =\frac{k_{0} m}{2 \pi} \int_{0}^{\theta} \frac{1}{x(\theta-x)^{1 / 2}} b \exp \left[-\frac{b^{2}}{4}\left(\theta-2 m+\frac{m^{2}}{x}\right)\right] d x,
\end{aligned}
$$


where $m=a / b$. (Recall that $m<\theta$.)

Suppose first that $m \leqslant 3 \theta / 4$. Using the inequality

$$
b \exp \left[-\frac{b^{2}}{4}\left(\theta-2 m+\frac{m^{2}}{x}\right)\right] \leqslant \frac{(2 x)^{1 / 2}}{\left(m^{2}+x(\theta-2 m)\right)^{1 / 2}} \exp \left(-\frac{1}{2}\right),
$$

we get

$$
J_{2} \leqslant \frac{k_{0} m \exp [-(1 / 2)]}{\sqrt{2} \pi} \int_{0}^{\theta} \frac{d x}{x^{1 / 2}(\theta-x)^{1 / 2}\left(m^{2}+x(\theta-2 m)\right)^{1 / 2}} .
$$

Since

$$
m^{2}+x(\theta-2 m) \geqslant \min \left(m^{2},(\theta-m)^{2}\right) \geqslant \min \left\{m^{2},(\theta / 4)^{2}\right\},
$$

$$
J_{2} \leqslant \frac{3}{\sqrt{2}} k_{0} \exp \left(-\frac{1}{2}\right) \text { if } m \leqslant \frac{3 \theta}{4} \text {. }
$$

If $m>3 \theta / 4$, we first consider

$$
J_{2}^{\prime} \equiv \int_{0}^{\theta / 2} \frac{1}{x(\theta-x)^{1 / 2}} b \exp \left[-\frac{b^{2}}{4}\left(\theta-2 m+\frac{m^{2}}{x}\right)\right] d x
$$

$$
\begin{aligned}
& <\int_{0}^{\theta / 2} \frac{1}{x(\theta-x)^{1 / 2}} b \exp \left(-\frac{b^{2} m^{2}}{8 x}\right) \exp \left[-\frac{b^{2}}{4 \theta}(\theta-m)^{2}\right] d x \\
& <\int_{0}^{\theta / 2} \frac{1}{x(\theta-x)^{1 / 2}} b \exp \left(-\frac{b^{2} \theta^{2}}{32 x}\right) d x
\end{aligned}
$$

Since

$$
\begin{gathered}
b \exp \left(-\frac{b^{2} \theta^{2}}{32 x}\right) \leqslant \frac{4 x^{1 / 2}}{\theta} \exp \left(-\frac{1}{2}\right), \\
J_{2}^{\prime} \leqslant \frac{1}{(\theta / 2)^{1 / 2}} \int_{0}^{\theta / 2} \frac{d x}{\sqrt{x}} \cdot \frac{4}{\theta} \exp \left(-\frac{1}{2}\right) \leqslant \frac{8}{\theta} \exp \left(-\frac{1}{2}\right) .
\end{gathered}
$$

Consider next

(A.12) $J_{2}^{\prime \prime} \equiv \int_{\theta / 2}^{\theta} \frac{1}{x(\theta-x)^{1 / 2}} b \exp \left[-\frac{b^{2}}{4}\left(\theta-2 m+\frac{m^{2}}{x}\right)\right] d x$.

Since $m>3 \theta / 4$,

$$
\theta-2 m+\frac{m^{2}}{x}=\frac{(\theta-m)^{2}+(2 m-\theta)(\theta-x)}{x} \geqslant \frac{1}{2}(\theta-x) .
$$

Hence 
(A.14) $\quad J_{2}^{\prime \prime} \leqslant \frac{2}{\theta} \int_{\theta / 2}^{\theta} \frac{1}{(\theta-x)^{1 / 2}} b \exp \left[-\frac{b^{2}}{8}(\theta-x)\right] d x \leqslant \frac{2}{\theta} 2 \sqrt{2 \pi}$.

From (A.10)-(A.12) and (A.14) it follows that

$$
J_{2} \leqslant 2 k_{0}\left[\frac{\sqrt{2}}{\sqrt{\pi}}+\frac{2}{\pi} \exp \left(-\frac{1}{2}\right)\right] \text { if } m>\frac{3 \theta}{4} .
$$

Combining this with (A.9) we get

$$
J_{2} \leqslant 2 k_{0}\left[\frac{\sqrt{2}}{\sqrt{\pi}}+\frac{2}{\pi} \exp \left(-\frac{1}{2}\right)\right]
$$

It remains to evaluate $J_{3}$. Clearly,

$$
J_{3}=\frac{k_{0}}{\pi} \int_{0}^{\theta} \frac{1}{x^{1 / 2}(\theta-x)^{1 / 2}} b \exp \left[-\frac{b^{2}}{4}\left(\theta-2 m+\frac{m^{2}}{x}\right)\right] d x .
$$

Suppose first that $m<3 \theta / 4$. Then

$$
\begin{aligned}
J_{3} & \leqslant \frac{k_{0}}{\pi} \int_{0}^{\theta} \frac{1}{x^{1 / 2}(\theta-x)^{1 / 2}} b \exp \left[-\frac{b^{2}}{4}(\theta-m)^{2}\right] d x \\
& \leqslant \frac{k_{0}}{\pi} \int_{0}^{\theta} \frac{1}{x^{1 / 2}(\theta-x)^{1 / 2}} b \exp \left[-\frac{b^{2} \theta}{16}\right] d x \leqslant \frac{2 k_{0}}{\sqrt{\theta}} \exp \left(-\frac{1}{2}\right) .
\end{aligned}
$$

If $m>3 \theta / 4$, we first consider

$$
J_{3}^{\prime} \equiv \int_{0}^{\theta / 2} \frac{1}{x^{1 / 2}(\theta-x)^{1 / 2}} b \exp \left[-\frac{b^{2}}{4}\left(\theta-2 m+\frac{m^{2}}{x}\right)\right] d x
$$

$$
\begin{aligned}
& \leqslant \int_{0}^{\theta / 2} \frac{1}{x^{1 / 2}(\theta-x)^{1 / 2}} b \exp \left[-\frac{b^{2} \theta}{32 x}\right] d x \\
& \leqslant \frac{4 \exp (-1 / 2)}{\theta} \int_{0}^{\theta / 2} \frac{d x}{(\theta-x)^{1 / 2}} \leqslant \frac{8 \exp (-1 / 2)}{\theta^{1 / 2}} .
\end{aligned}
$$

If $m \leqslant 3 \theta / 4$ then we have, by (A.13),

$$
\begin{aligned}
J_{3}^{\prime \prime} & \equiv \int_{\theta / 2}^{\theta} \frac{1}{x^{1 / 2}(\theta-x)^{1 / 2}} b \exp \left[-\frac{b^{2}}{4}\left(\theta-2 m+\frac{m^{2}}{4}\right)\right] d x \\
& \leqslant\left(\frac{2}{\theta}\right)^{1 / 2} \int_{\theta / 2}^{\theta} \frac{1}{(\theta-x)^{1 / 2}} b \exp \left[-\frac{b^{2}}{8}(\theta-x)\right] d x \\
& \leqslant \frac{2 \sqrt{2 \pi} \sqrt{2}}{\theta^{1 / 2}}=\frac{4 \sqrt{\pi}}{\theta^{1 / 2}} .
\end{aligned}
$$

Combining the estimates (A.16), (A.17) we find that 


$$
J_{3} \leqslant \frac{4 k_{0}}{\pi^{1 / 2} \theta^{1 / 2}}\left[1+\frac{2}{\pi^{1 / 2}} \exp \left(-\frac{1}{2}\right)\right] \quad \text { if } m>\frac{3 \theta}{4} .
$$

In view of (A.16), the last inequality is satisfied also in case $m \leqslant 3 \theta / 4$. Combining this inequality with (A.15), (A.8), and using (A.7), the assertion (A.6) follows.

ADDED IN PROOF. (1) It was pointed out by Robert Jensen that the estimate (4.42) can be derived without recourse to Lemma 4.4. Instead of working with the function $w$ introduced in Lemma 4.4, we work with

$$
w(\xi, \tau)=k_{0}\left\{\exp \left[-b(\xi-\alpha+a)+b^{2}(\tau-\sigma)\right]-1\right\} .
$$

(2) In a recent paper Smoothness of the free boundary in the Stefan problem with supercooled water, Robert Jensen proved the existence of a global solution for the Stefan problem corresponding to the variational inequality

$$
\begin{aligned}
& u \geqslant 0, \quad\left(u_{t}-u_{x x}\right)(v-u) \geqslant-(v-u) \text { for every } v \geqslant 0, \\
& u(x, 0)=h(x)
\end{aligned}
$$

provided $h^{\prime}(x)$ changes sign just once. His method can be applied to extend Theorem 5.2 (beyond the last remark of $\$ 5$ ) to the case when no restrictions are made on the number of sign changes of the function $g(x)$ (defined in (5.43)).

\section{REFERENCES}

1. A. Bensoussan and A. Friedman, Nonlinear variational inequalities and differential games with stopping times, J. Functional Analysis 16 (1974), 305-352. MR 50 \#6531.

2. A. Bensoussan, M. Goursat and J. L. Lions, Contrôle impulsionnel et inéquations quasivariationnelles stationnaires, C. R. Acad. Sci. Paris Sér. A-B 276 (1973), A1279-A1284. MR 47 \#5691.

3. A. Bensoussan and J. L. Lions, Problèmes de temps d'arrèt optimal et inéquations variationelles paraboliques, Applicable Anal. 3 (1973), 267-295.

4. Nouvelles méthodes en contrôle impulsionnel, J. Appl. Math. and Optimization 1 (1975), 289-312.

5. Acad. Sci. Paris Sér. A-B 276 (1973), A1189-A1192. MR 47 \#5690.

6. Contrôle impulsionnel et inéquations quasi-variationnelles d'evolution, C. R. Acad. Sci. Paris Sér. A-B 276 (1973), A1333-A1338. MR 47 \#5692.

7. Contrôle impulsionnel et systèmes d'inéquations quasi-variationnelles, C. R. Acad. Sci. Paris Sér. A 278 (1974), 747-751. MR 49 \#5996.

8. Inéquations quasi-variationnelles décroisantes, Congrès d'Analyse Convexe (St. Pierre de Chartieuse, 1974), Lecture Notes in Math., Springer-Verlag,Berlin and New York (to appear).

9. H. Brezis, Problemes unilatéraux, J. Math. Pures Appl. 51 (1972), 1-168.

10. H. Brezis and A. Friedman, Estimates on the support of solutions of parabolic variational inequalities, Illinois J. Math. 20 (1976), 82-97.

11. G. Duvaut, Résolution d'un problème de Stefan (fusion d'un bloc de glace à zéro degré), C. R. Acad. Sci. Paris Sér. A-B. 276 (1973), A1461-A1463. MR 48 \#6688. 
12. A. Friedman, Free boundary problems for parabolic equations. I. Melting of solids, J. Math. and Mech. 8 (1959), 499-517. MR 26 \# 1626.

13. _ Partial differential equations of parabolic type, Prentice-Hall, Englewood Cliffs, N. J., 1964. MR 31 \#6062.

14. Stochastic games and variational inequalities, Arch. Rational Mech. Anal. 51 (1973), 321-346. MR 50 \#4059.

15. __ Regularity theorem for variational inequalities in unbounded domains and applications to stopping time problems, Arch. Rational Mech. Anal. 52 (1973), 134-160. MR 50 \# 5596.

16. __ Parabolic variational inequalities in one space dimension and smoothness of the free boundary, J. Functional Analysis 18 (1975), 151-176.

17. The shape and smoothness of the free boundary for some elliptic variational inequalities, Indiana Univ. Math. J. 25 (1976), 103-118.

18. A problem in hydraulics with non-monotone free boundary, Indiana Univ. Math. J. 25 (1976), 577-592.

19. A. Friedman and D. Kinderlehrer, $A$ one phase Stefan problem, Indiana Univ. Math. J. 24 (1975), 1005-1035.

20. $\ldots$ A class of parabolic quasi-variational inequalities, J. Differential Equations 21 (1976), 395-416.

21. J.-L. Lions and G. Stampacchia, Variational inequalities, Comm. Pure Appl. Math. 20 (1967), 493-519. MR 35 \#7178.

22. V. A. Solonnikov, A priori estimates for second-order parabolic equations, Trudy Mat. Inst. Steklov. 70 (1964), 133-212; English transl., Amer. Math. Soc. Transl. (2) 65 (1967), 51-137. MR 28 \# 5267.

23. L. Tartar, Inéquations quasi variationnelles abstraites, C. R. Acad. Sci. Paris Sér. A 278 (1974), 1193-1196. MR 49 \#9703.

24. P. Van Moerbeke, An optional stopping time problem for linear reward, Acta Math. 132 (1974), 1-41.

Departement de Mathematiques, Universite de Paris IX, 75016 Paris, France

INSTItUt DE Recherche D'INFormatique et D'Automatique (IRIA), 78150 Le Chesnay, France

Department of Mathematics, Northwestern University, Evanston, Illinois 60201 